\title{
Localization Formulas, Superconnections, and the Index Theorem for Families
}

\author{
J.-M. Bismut \\ Département de Mathématiques, Université Paris-Sud, F-91405 Orsay, France
}

\begin{abstract}
In this paper, we give a new proof of the localization formulas of Berline and Vergne [9] and Duistermaat and Heckman [18]. When interpreted in the framework of Atiyah [2], the probabilistic heat equation proof of the Index Theorem given in our paper [12] appears as the rigorous infinite dimensional version of this new proof of the localization formulas in finite dimensions. The results of Quillen [25] on superconnections are briefly presented. The heat equation proofs [15] of the Index Theorem for families are described. It is shown that in this framework, the superconnections formalism is the operator theoretic description of integration along the fiber in the loop space.
\end{abstract}

\section{Table of Contents}

Introduction . . . . . . . . . . . . . . . . . . . . . . . . . . . . 128

I. Localization Formulas in Equivariant Cohomology . . . . . . . . . . . . 130

a) Assumptions and Notations . . . . . . . . . . . . . . . . . . . . . . 131

b) A Gaussian Proof of a Formula of Berline and Vergne and Duistermaat and Heckman.

c) The Case of Two Commuting Vector Fields

d) Integration Along the Fiber and Localization . . . . . . . . . . . . . 137

II. The Probabilistic Proof of the Index Theorem . . . . . . . . . . . . . . 141

a) Assumptions and Notations . . . . . . . . . . . . . . . . . . . . . . 142

b) The Loop Space and the Action of $S_{1}$. . . . . . . . . . . . . . . . . . 142

c) The Index of the Dirac Operator . . . . . . . . . . . . . . . . . . . . . 143

d) Equivariant Cohomology on the Loop Space and the Index Theorem . . . . 144

e) The Heat Equation as a Natural Proof of Localization in Infinite Dimensions. . 145

f) The Infinitesimal Lefschetz Formulas . . . . . . . . . . . . . . . . 148

III. Integration Along the Fiber in the Loop Space, and the Index Theorem for Families 150

a) The Construction by Quillen of the Chern Character of a Difference Bundle . . 151

b) Elementary Properties of a Fibered Manifold. . . . . . . . . . . . . . 152

c) A Connection on an Infinite Dimensional Bundle . . . . . . . . . . . . . 153

d) The Dirac Operator in the Fibers . . . . . . . . . . . . . . . . . . 154 
e) Index Theory and Infinite Dimensional Chern-Weil Theory . . . . . . . . 154

f) The Levi-Civita Superconnection . . . . . . . . . . . . . . . . . . 155

g) The Index Theorem for Families: A First Proof. . . . . . . . . . . . . 156

h) A Second Proof . . . . . . . . . . . . . . . . . . . . . . . . 159

i) Superconnections, Quillen's Formalism, and Integration Along the Fiber in the Loop Space .

\section{Introduction}

In this paper, we want to develop some relations of Index Theory to the equivariant cohomology of the loop space.

Let us recall that in [2], Atiyah, describing an idea of Witten, had shown that at least formally, the Index of the Dirac operator acting on the spin complex of a spin manifold $M^{0}$ could be written as the integral over the loop space $M$ of a differential form which is equivariantly closed (in the sense of Berline and Vergne [9] and Witten [26]), with respect to the action of $S_{1}$ on $M$ by rotations. By applying formally a localization formula of Berline and Vergne [9], Duistermaat and Heckman [18], Atiyah showed that the local Index formula could be obtained in this situation.

In [13], we extended Atiyah's formalism to the case of a twisted spin complex. We proved that the same formal result was still true when replacing the spin complex by a twisted spin complex. In particular, the natural lift to $M$ of the Chern character of a bundle $\xi$ over $M^{0}$ appears naturally as the characteristic class associated with an infinite dimensional bundle whose structure group is a KacMoody group.

Reviewing the main aspects of localization formulas in equivariant cohomology and $K$-theory, Atiyah and Bott [4] suggested that a direct proof of localization in infinite dimensions should be given. The differential geometric proofs of Berline and Vergne [9], Duistermaat and Heckman [18], seem to be difficult to adapt in infinite dimensions, because they make use of Stokes formula to equal an integral over the whole space with an integral over a small sphere. As is well known, spheres have pathologies in infinite dimensions.

Our first purpose is to show that the well known heat equation method in Index Theory, which was introduced by McKean and Singer [24] and Atiyah et al. [5], when correctly interpreted in the loop space via Brownian motion, is by itself a rigorous proof in infinite dimensions of a localization formula in equivariant cohomology on the loop space. Essentially, we give another proof in finite dimensions of the localization formulas of $[9,18]$. The heat equation method, when interpreted probabilistically, appears naturally as the obvious analogue in infinite dimensions of this new proof. The merit of this proof is that it reproduces in a finite dimensional situation the main steps of the probabilistic proof of the Index Theorem of Atiyah-Singer which we gave in [12], inclusively in its intermediary computations.

In [14], now inspired by the finite dimensional new proof, we gave a direct heat equation proof of the infinitesimal Lefschetz formulas of Atiyah and Singer [6]. We also refer to Berline and Vergne [27] for the application of such formulas to the orbital formulas of Kirillov. 
We were then interested in finding a heat equation proof of the Index Theorem for families of Dirac operators (see Atiyah and Singer [7]) not in the $K$-theoretic sense, but only in the sense of rational cohomology. Our idea was that Atiyah's formalism could also be extended in this case, replacing an integral on the loop space by integration along the fiber in a fibered loop space.

Now there is an obvious "integration along the fiber" version of localization in equivariant cohomology. In the manner of Atiyah [2], we first checked that formally, we obtained the Index Theorem for families of Dirac operators. We then had to make a rigorous proof of this formalism. Recall that in infinite dimensions, there is still no natural cohomological formalism, and no Poincaré duality. So we first wrote a differential geometric proof of localization with "integration along the fiber" inspired from our initial simple proof of localization.

On the other hand, by using superconnections, Quillen [25] had given a construction of the Chern character of a finite dimensional difference bundle $E_{0}-E_{1}$ when the $Z_{2}$ graded bundle $E=E_{0} \oplus E_{1}$ is endowed with an "odd" linear mapping $D$ exchanging $E_{0}$ and $E_{1}$. Quillen was explicitly thinking of extending his formalism to an infinite dimensional situation.

In [15], we succeeded in extending Quillen's formalism to infinite dimensions. More precisely, we showed, using pseudodifferential techniques, that the "heat equation" analogue of Quillen's formulas for the Chern character of a finite dimensional difference bundle was in fact the Chern character of $\operatorname{Ker} D-\operatorname{Coker} D$. Translating this formalism on the loop space, we found that our heat equation formula was the integration along the fiber version we were looking for. Inspired by the finite dimensional proof of localization, we could then choose the right superconnection to obtain the Index Theorem for families. We also obtained a second proof based on a slightly different principle.

In this paper, we describe in detail the new differential geometric proofs of localization in equivariant cohomology. We analyse our probabilistic proofs of the Index Theorem, of the infinitesimal Lefschetz formulas, and of the Index Theorem for families, and we show how in this context, the $Z_{2}$ gradation formalism is the natural way by which operator theory calculates integration along the fiber in the loop space.

We do not review in more detail the papers of Alvarez-Gaumé [1], Berline and Vergne [8], Friedan and Windey [19], and Getzler [20,21] which contain different proofs of the Index Theorem for Dirac operators. Let us just point out that in this framework, because of the intimate relationship of the loop space to operator theory, any statement in the loop space has a natural translation in operator theory, so that all the direct proofs of the classical Index Theorem are intimately connected with probability. In particular, the computations in Getzler [21] have a direct probabilistic interpretation, in terms of certain stochastic area formulas of P. Lévy, as should clearly appear in [12]. Also the evaluation of the $\hat{A}$ polynomial as related to the determinant of the exponential mapping in $\mathrm{SO}(n)$ - which is crucial in Berline and Vergne [8] - has a natural probabilistic interpretation since classically (see [10, Sect. 4]) such determinants can be expressed in terms of path integrals. On the other hand, it seems that the supersymmetric arguments in Alvarez-Gaumé [1] and Friedan and Windey [19] could be translated in the language of the equivariant cohomology of the loop space. 
Our paper is organized as follows. In Sect. 1, we prove localization formulas in equivariant cohomology in finite dimensions. The proofs are given in more detail, or are even longer than necessary, because we need to use objects which can be extended in infinite dimensions in an obvious way.

In Sect. 2, we analyse the probabilistic proofs of the Index Theorem [12], and of the infinitesimal Lefschetz formulas [14] in the light of Sect. 1.

Section 3 describes our proofs of the Index Theorem for families and also discusses their relations with Sect. 1.

\section{Localization Formulas in Equivariant Cohomology}

In this section, we give an expanded treatment of the localization formulas of Berline and Vergne [9] and Duistermaat and Heckman [18]. As pointed out in the Introduction, our purpose is not to give another proof of these results, but to build a proof which has two qualities:

- It is very simple in finite dimensions.

- It has the property that our heat equation proofs of

- the Index Theorem of Atiyah and Singer [12],

- the infinitesimal Lefschetz formulas [14],

- the Index Theorem of Atiyah and Singer for families [15]

reproduce in detail the various technical steps of this proof in finite dimensions.

It is mostly based on the fact that if $X$ is a Killing vector field on a Riemannian manifold, if $X$ is identified with a 1 form by the metric, as $t \downarrow \downarrow 0$ the Gaussian shaped differential form $\exp \left\{-\frac{d X+|X|^{2}}{2 t}\right\}$ produces the desired localization on $(X=0)$. Let us point out that Quillen [25] and Mathai and Quillen [23] have also introduced Gaussian shaped differential forms to study problems related with localization.

In a), we introduce the main assumptions and notations.

In b), we give two proofs of the localization formula of Berline and Vergne [9] and Duistermaat and Heckman [18]. The first proof is closely related in spirit to Berline and Vergne [9], Duistermaat and Heckman [18], although it uses the previously mentioned Gaussian shaped form. As we shall see in Sect. 2, b) is useful to understand the main steps of the proof [12] of the Index Theorem.

In c), we prove a localization formula with two commuting Killing vector fields. We will verify in Sect. 2 that c) is the exact model for our proof of the infinitesimal Lefschetz formulas [14].

In d), we give two proofs of an integration along the fiber version of the localization formulas. As we shall see in Sect. 3, these two proofs are the models for our two proofs of the Index Theorem for families in [15].

Since we need to produce proofs which have infinite dimensional analogues, some proofs are more developed than needed in finite dimensions. 


\section{a) Assumptions and Notations}

$M$ is a $C^{\infty}$ connected compact oriented Riemannian manifold of dimension $n$. $X$ is a Killing vector field on $M . \Lambda(M)$ is the algebra of $C^{\infty}$ differential forms over $M$, which splits into

$$
\Lambda(M)=\bigoplus_{0}^{n} \Lambda^{p}(M)
$$

where $\Lambda^{p}(M)$ is the set of $p$-forms.

The exterior differentiation operator $d$ and the interior product operator $i_{X}$ send $\Lambda(M)$ into itself.

Following Berline and Vergne [9], Witten [26], we set the following definition.

Definition 1.1. $\mu \in \Lambda(M)$ is said to be $X$ equivariantly closed if:

$$
\left(d+i_{X}\right) \mu=0 \text {. }
$$

Let us recall that if $B$ is an oriented submanifold of dimension $p$, if $\mu=\mu^{0}+\ldots+\mu^{n}$, with $\mu^{i}$ of degree $i$, then by definition

$$
\int_{B} \mu=\int_{B} \mu^{p}
$$

Definition 1.2. $M^{X}$ is the submanifold

$$
M^{X}=\{x \in M ; X(x)=0\} .
$$

$N$ is the normal bundle of $M^{X}$ in $M$, and $\pi$ is the projection $N \rightarrow M^{X}$.

For $x \in M^{X}$, we have

$$
T_{x} M=T_{x} M^{X} \oplus N_{x}
$$

b) A Gaussian Proof of a Formula of Berline and Vergne and Duistermaat and Heckman

Let $J_{X}$ be the infinitesimal action of $X$ in $N$. $J_{X}$ is an antisymmetric element of End $N$ which defines a non-degenerate 2-form on $N Y, Y^{\prime} \in N \rightarrow\left\langle Y, J_{X} Y^{\prime}\right\rangle . N$ is then naturally oriented. Since $M$ is oriented, it follows that $M^{X}$ has a natural orientation. Also, if $A \in \operatorname{End}(N)$ is antisymmetric, the Pfaffian $\operatorname{Pf}(A)$ is welldefined.

$M^{X}$ is totally geodesic in $M$. It then follows from (1.2) that if $\nabla$ is the Levi-Civita connection of $T M, \nabla$. induces an Euclidean connection on $N$.

If $R$ is the curvature tensor of $M$, for $x \in M^{X}, Y, Z \in T_{x} M^{X}, N_{x}$ is stable under $R_{x}(Y, Z)$, and $R_{x}(Y, Z)$ commutes with $J_{X}$.

In what follows the Pfaffian $\operatorname{Pf}\left[\frac{J_{X}+R}{2 \pi}\right]$ of $\frac{J_{X}+R}{2 \pi}$ is taken as the Pfaffian of antisymmetric matrices acting on $N$.

We now prove the result of Berline and Vergne [9], Duistermaat and Heckman [18].

Theorem 1.3. If $\mu \in \Lambda(M)$ is $X$ equivariantly closed, then

$$
\int_{M} \mu=\int_{M^{X}} \frac{\mu}{\operatorname{Pf}\left[\frac{J_{X}+R}{2 \pi}\right]} .
$$


Proof. We give two proofs.

Proof No. 1. This proof uses a linearization technique which also appears in [9, 18]. However, the localization is obtained using a different method.

For any $y \in N$, using the connection $\nabla$. restricted to $N$, we can split $T N$ into

$$
T N=T^{H} N \oplus T^{V} N
$$

$T^{V} N$ is made of vectors living in the fiber $N$, and $T^{H} N$ corresponds to the parallel displacement of $y \in N$ in the "horizontal" directions. For $Y \in T N, Y^{H}, Y^{V}$ denotes the components of $Y$ according to the splitting (1.4).

Also using the exponential mapping, we can identify a tubular neighborhood of $M^{X}$ in $M$ with a neighborhood $V$ of $M^{X}$ (considered as the zero section of $N$ ) in $N$. Using this identification, for $y \in V$, we have

$$
X(y)=J_{X} y .
$$

Let $\alpha$ be the one form on $N$

$$
Y \in T N \rightarrow \alpha(Y)=\left\langle J_{X} y, Y^{V}\right\rangle .
$$

It is easy to verify that if $Y, Z \in T N$,

$$
d \alpha(Y, Z)=2\left\langle J_{X} Y^{V}, Z^{V}\right\rangle+\left\langle R_{\pi y}\left(\pi_{*} Y, \pi_{*} Z\right) y, J_{X} y\right\rangle .
$$

Then for $y \in V$,

$$
\alpha(X)=|X|^{2}
$$

Also we identify $X$ to a 1 -form using the metric of $M$. By using a partition of unity argument, we can find a $C^{\infty}$ one form $\beta$ on $T M$ having the following properties.

- It coincides with $\alpha$ on a neighborhood $V^{\prime} \subset V$ of $M^{X}$ in $M$.

- Also

$$
\beta(X)=|X|^{2}
$$

Let $T$ be a maximal torus in the group of isometries of $M$ such that $X$ is contained in its Lie algebra $t$. By averaging $\beta$ over $T$, we find that we can also assume that $\beta$ is $T$ invariant so that

$$
L_{X} \beta=0 .
$$

Since $L_{X}=\left(d+i_{X}\right)^{2}$, it follows that

$$
\left.\left(d+i_{X}\right)\left[\left(d+i_{X}\right) \beta\right)\right]=0 .
$$

We claim that for any $s \geqq 0$,

$$
\int_{M} \mu=\int_{M} \exp \left\{-s\left(d+i_{X}\right) \beta\right\} \mu .
$$

To prove (1.10), we note that

$$
\frac{\partial}{\partial s} \int_{M} \exp \left\{-s\left(d+i_{X}\right) \beta\right\} \mu=-\int_{M}\left(d+i_{X}\right) \beta \exp \left\{-s\left(d+i_{X}\right) \beta\right\} \mu .
$$


Also the assumption on $\mu$ and (1.9) show that

$$
\begin{aligned}
\left(d+i_{X}\right) \mu & =0, \\
\left(d+i_{X}\right)\left[\exp \left\{-s\left(d+i_{X}\right) \beta\right]\right. & =0,
\end{aligned}
$$

and so (1.11) is equal to

$$
-\int_{M}\left(d+i_{X}\right)\left[\beta \exp \left\{-s\left(d+i_{X}\right) \beta\right\} \mu\right]=0 .
$$

The right-hand side of (1.10) is constant in $s$. For $s=0$, equality holds in (1.10) so that (1.10) has been proved.

For $t>0$, we have found that

$$
\int_{M} \mu=\int_{M} \exp \left\{-\frac{d \beta}{2 t}-\frac{|X|^{2}}{2 t}\right\} \mu .
$$

As $t \downarrow \downarrow 0$, since $X \neq 0$ out of $M^{X}$,

$$
\int_{M} \exp \left\{-\frac{d \beta}{2 t}-\frac{|X|^{2}}{2 t}\right\} \mu \sim \int_{V^{\prime}} \exp \left\{-\frac{d \beta}{2 t}-\frac{|X|^{2}}{2 t}\right\} \mu .
$$

We identify $J_{X}$ with the 2-form on $T N$

$$
Y, Z \rightarrow\left\langle Y^{V}, J_{X} Z^{V}\right\rangle
$$

and $R$ with the 2-form

$$
Y, Z \rightarrow R_{\pi y}\left(\pi_{*} Y, \pi_{*} Z\right) .
$$

Using (1.7), (1.8) we find that the right-hand side of (1.15) is equal to

$$
\int_{V^{\prime}} \exp \left\{\frac{J_{X}}{t}-\frac{\left\langle R_{\pi y}(\cdot, \cdot) y, J_{X} y\right\rangle}{2 t}\right\} \exp \left\{-\frac{\left|J_{X} y\right|^{2}}{2 t}\right\} \mu_{y} .
$$

We now do the computations on one given connected component of $M^{X}$. Let $n^{\prime}$ be the dimension of $N$ (which is even).

By making the change of variables $y=\sqrt{t} y^{\prime}$, we find that (1.18) is asymptotically equal to

$$
t^{n^{\prime} / 2} \int_{N} \exp \left\{\frac{J_{X}}{t}-\frac{\left\langle R_{x}(\cdot, \cdot) y, J_{X} y\right\rangle}{2}\right\} \exp \left\{-\frac{\left|J_{X} y\right|^{2}}{2}\right\} \mu_{y \sqrt{t}} .
$$

By expanding $\exp \frac{J_{X}}{t}$, we find that the only term which is not killed by $t^{n^{\prime} / 2}$ is

$$
\frac{\left(\frac{J_{X}}{t}\right)^{n^{\prime} / 2}}{\left(\frac{n^{\prime}}{2}\right) !}=\left(\operatorname{Pf} J_{X}\right) d y
$$

where $d y$ is the volume form of $N$ which defines the orientation of $N$. On the other hand, the vertical components of $\mu$ are killed as $t \downarrow \downarrow 0$. So we find that the limit of 
(1.19) is

$$
\int_{M^{X}}\left[\int_{N_{x}} \exp \left\{-\frac{\left\langle R_{x}(\cdot, \cdot) y, J_{X} y\right\rangle}{2}-\frac{\left|J_{X} y\right|^{2}}{2}\right\}\left(\operatorname{Pf} J_{X}\right) d y\right] \mu .
$$

Let $R^{\prime} \in$ End $N_{x}$ be antisymmetric and assume it commutes with $J_{X}$. Then $J_{X} R^{\prime}$ is symmetric. It follows that if $R^{\prime}$ has a norm which is small enough,

$$
\int_{N_{x}} \exp \left\{-\frac{\left\langle R^{\prime} y, J_{X} y\right\rangle}{2}-\frac{\left|J_{X} y\right|^{2}}{2}\right\} d y=\frac{(2 \pi)^{n^{\prime} / 2}}{\left(\operatorname{det}\left[-J_{X}^{2}-J_{X} R^{\prime}\right]\right)^{1 / 2}} .
$$

Since $n^{\prime}$ is even, we have

$$
\operatorname{det}\left(-J_{X}^{2}-J_{X} R^{\prime}\right)=\left(\operatorname{det} J_{X}\right) \operatorname{det}\left(J_{X}+R^{\prime}\right) .
$$

Also recall that $\operatorname{det} R^{\prime}=\left(\operatorname{Pf} R^{\prime}\right)^{2}$.

Since (1.22) is analytic in $R^{\prime}$ for $\left|R^{\prime}\right|$ small enough, we find

$$
\frac{\operatorname{Pf} J_{X}}{\operatorname{det}\left(-J_{X}^{2}-J_{X} R^{\prime}\right)^{1 / 2}}=\frac{1}{\operatorname{Pf}\left[J_{X}+R^{\prime}\right]} .
$$

Recall that $\exp \left\{-\frac{\left\langle R(\cdot, \cdot) y, J_{X} y\right\rangle}{2}\right\}$ is a finite power series in the variable $R$, so we can use (1.22), (1.23) with $R(\cdot, \cdot)$ instead of $R^{\prime}$. Equation (1.3) is proved.

Proof No. 2. We identify $X$ with a 1 -form by the Riemannian metric. Since $X$ is a Killing vector field, as a one form, $X$ is $L_{X}$ invariant so that

$$
\left(d+i_{X}\right)\left[\left(d+i_{X}\right) X\right]=0 .
$$

As in (1.14), we find for $t>0$,

$$
\int_{M} \mu=\int_{M} \exp \left\{-\frac{\left(d+i_{X}\right) X}{2 t}\right\} \mu .
$$

As in (1.15), we can prove that as $t \downarrow \downarrow 0$, the right-hand side of (1.25) is asymptotically equal to

$$
\int_{V} \exp \left\{-\frac{\left(d+i_{X}\right) X}{2 t}\right\} \mu .
$$

Also it is trivial that if $Y, Z \in T M$,

$$
d X(Y, Z)=2\left\langle\nabla_{Y} X, Z\right\rangle .
$$

Note that on $M^{X}, J_{X}$ and $\nabla . X$ coincide on $N$. Also since $\nabla$. is $X$ invariant, it can be easily proved (see Berline and Vergne [9]), that

$$
\left[\nabla_{Y}(\nabla . X)\right]+R(X, Y)=0 \text {. }
$$

We now evaluate (1.25). Take $x \in M^{X}, U$ an open ball in $M^{X}$ such that $N_{U} \simeq U \times R^{n}$. We also assume that for $\varepsilon>0$ small enough, $\left\{(x, y) \in N_{U} ;|y| \leqq \varepsilon\right\}$ has been identified with a neighborhood of $U$ in $M$ by a diffeomorphism $f_{U}$ which sends $(x, 0) \in N_{U}$ into $x \in U$, whose differential $d f_{U}$ is the identity mapping at $(x, 0)$ : a natural candidate is the exponential mapping but other choices are also possible. 
As $t \downarrow \downarrow 0$, by making the change of variables $y=\sqrt{t} y^{\prime}$,

$$
\begin{aligned}
\int_{V^{\prime} \cap(x \in U)} \exp & -\left\{\frac{d X+|X|^{2}}{2 t}\right\} \mu \sim t^{\frac{n^{\prime}}{2}} \int_{U \times R^{\mathbf{n}^{\prime}}} \exp -\left\{\frac{d X(x, \sqrt{t} y)}{2 t}\right. \\
+ & \left.\frac{|X|^{2}(x, \sqrt{t} y)}{2 t}\right\} \mu(x, \sqrt{t} y) .
\end{aligned}
$$

Also since

$$
\left(\nabla_{y} X\right)(x, 0)=J_{X} y
$$

we find easily that

$$
\frac{|X|^{2}(x, \sqrt{t} y)}{2 t} \rightarrow \frac{\left|J_{X} y\right|^{2}}{2}
$$

If $Y, Z$ are taken among the vector fields $\frac{\partial}{\partial x^{i}}, \frac{\partial}{\partial y^{\alpha}}$, if $D$ denotes the covariant derivative operator for the Levi-Civita connection along the path $(x, \sqrt{t} y)$, we find using (1.27)

$$
\begin{aligned}
& \frac{(d X)(x, \sqrt{t} y)(Y, Z)}{2 t} \\
& =\frac{\left\langle J_{X} Y, Z\right\rangle}{t}-\frac{\left\langle R\left(J_{X} y, y\right) Y, Z\right\rangle}{2}+\left[\left\langle J_{X} D Y, Z\right\rangle+\left\langle J_{X} Y, D Z\right\rangle\right] \frac{1}{\sqrt{t}} \\
& \quad+\frac{1}{2}\left[\left\langle J_{X} D^{2} Y, Z\right\rangle+2\left\langle J_{X} D Y, D Z\right\rangle+\left\langle J_{X} Y, D^{2} Z\right\rangle\right]+O(t) .
\end{aligned}
$$

Because of the factor $t^{n^{\prime} / 2}$ in (1.28), it should be clear that in the limit, only those monomials in the vertical form $d y^{1} \ldots d y^{n^{\prime}}$ should be kept whose weight is exactly $\frac{1}{t^{n^{\prime} / 2}}$. It follows that in the expression

$$
\left(\left\langle J_{X} D Y, Z\right\rangle+\left\langle J_{X} Y, D Z\right\rangle\right) \frac{1}{\sqrt{t}}
$$

a non-zero contribution could appear only if one of the $Y, Z$ is in the family $\frac{\partial}{\partial x^{i}}$, and the other in the family $\frac{\partial}{\partial y^{\alpha}}$. However, $J_{X} \frac{\partial}{\partial x^{i}}=0$. Similarly, in the term

$$
-\frac{\left\langle R\left(J_{X} y, y\right) Y, Z\right\rangle}{2}+\frac{1}{2}\left[\left\langle J_{X} D^{2} Y, Z\right\rangle+2\left\langle J_{X} D Y, D Z\right\rangle+\left\langle J_{X} Y, D^{2} Z\right\rangle\right]
$$

both $Y$ and $Z$ have to be in the family $\frac{\partial}{\partial x^{i}}$. This kills $\left\langle J_{X} D^{2} Y, Z\right\rangle+\left\langle J_{X} Y, D^{2} Z\right\rangle$, since $J_{X} \frac{\partial}{\partial x^{i}}=0$.

For $Y \in T_{(x, 0)} M^{X}$, set

$$
Y=Y^{H}+Y^{V} ; \quad Y^{H} \in T_{(x, 0)} M^{X}, \quad Y^{V} \in N_{(x, 0)} .
$$


As $t \downarrow \downarrow 0$, in (1.28), we should then replace $\frac{d X}{2 t}(x, \sqrt{t} y)$ by the 2-form

$$
\left\langle J_{X}\left(Y^{V}+D Y^{H}\right), Z^{V}+D Z^{H}\right\rangle-\frac{\left\langle R\left(J_{X} y, y\right) Y^{H}, Z^{H}\right\rangle}{2} .
$$

Since $J_{X}$ is non-degenerate on $N$, integrating in the vertical variables $y$ means that we can ignore $D Y^{H}, D Z^{H}$ in (1.33). Also the well-known symmetries of the LeviCivita tensor $R$ imply that

$$
\left\langle Y^{H}, R\left(J_{X} y, y\right) Z^{H}\right\rangle=\left\langle R\left(Y^{H}, Z^{H}\right) y, J_{X} y\right\rangle .
$$

We finally find that the limit of (1.33) is exactly

$$
\int_{M^{X}}\left[\int_{N} \exp \left\{J_{X}-\frac{\left\langle R(\cdot, \cdot) y, J_{X} y\right\rangle}{2}-\frac{\left|J_{X} y\right|^{2}}{2}\right\}\right] \mu,
$$

which coincides with (1.21).

Remark 1. The proof No. 1 is simpler than the proof No. 2. However, as we shall see, the proof No. 2 has two qualities.

- The aspects of the proofs of Berline and Vergne [9] and Duistermaat and Heckman [18] which were impossible to extend in infinite dimensions - namely the explicit use of Stokes formula - have disappeared.

- When correctly interpreted, the heat equation proof of the Index Theorem of Atiyah-Singer coincides with the proof No. 2.

Remark 2. If $M$ is non-compact, and $\mu$ has compact support, (1.3) still holds. If $\mu$ does not have compact support, (1.3) does not hold any more. However, under adequate growth conditions on $|X|$,

$$
\int_{M} \exp \left\{-\frac{\left(d+i_{X}\right) X}{2 t}\right\} \mu
$$

is still well-defined. A proof similar to (1.36) then shows that under adequate conditions, (1.36) does not depend on $t>0$. If an adequate control at infinity of the integral (1.36) is possible, Proof No. 2 will also show that for any $t>0$

$$
\int_{M} \exp \left\{-\frac{\left(d+i_{X}\right) X}{2 t}\right\} \mu=\int_{M^{X}} \frac{\mu}{\operatorname{Pf}\left[\frac{J_{X}+R}{2 \pi}\right]} .
$$

\section{c) The Case of Two Commuting Vector Fields}

Let $Y$ be another Killing vector field such that

$$
[X, Y]=0 \text {. }
$$

Equation (1.38) writes

$$
\nabla_{X} Y-\nabla_{Y} X=0
$$

Using (1.27), we find that if $x \in M^{X}, Z \in T_{x} M$,

$$
\nabla_{\nabla_{Z} X} Y-\nabla_{\nabla_{Z} Y} X=0 \text {. }
$$


The antisymmetric tensor $\nabla . Y$ commutes wuth $J_{X}$ on $M^{X} . N$ is then stable under $\nabla . Y$.

We now extend Theorem 1.3.

Theorem 1.4. If $\mu \in \Lambda(M) \otimes_{R} C$ is $X+i Y$ equivariantly closed, then

$$
\int_{M} \mu=\int_{M^{X}} \frac{\mu}{\operatorname{Pf}\left[\frac{J_{X}+i \nabla \cdot Y+R}{2 \pi}\right]} .
$$

Proof. As in the Proof No. 2 of Theorem 1.3, we can write that for any $t>0$

$$
\int_{M} \mu=\int_{M} \exp \left\{-\frac{\left(d+i_{X+i Y}\right)(X)}{2 t}\right\} \mu .
$$

Observe that

$$
\left|\exp -\frac{i\langle X, Y\rangle}{2 t}\right|=1
$$

so that as $t \downarrow \downarrow 0$, the integral (1.42) localizes as in Theorem 1.3. Also, using the notations of the Proof No. 2 of Theorem 1.3, we have

$$
\frac{\partial}{\partial \sqrt{t}}\langle X, Y\rangle(x, \sqrt{t} y)=\left(\left\langle\nabla_{y} X, Y\right\rangle+\left\langle X, \nabla_{y} Y\right\rangle\right)(x, \sqrt{t} y) \text {. }
$$

Using (1.39), we have for $t=0$

$$
\left\langle\nabla_{y} X, Y\right\rangle=-\left\langle\nabla_{Y} X, y\right\rangle=-\left\langle\nabla_{X} Y, y\right\rangle=0 .
$$

At $t=0,(1.43)$ is 0 . Also at $t=0$, using (1.27) and (1.39) again, we have:

$$
\begin{aligned}
\frac{\partial^{2}}{\partial(\sqrt{t})^{2}}\langle X, Y\rangle(x, \sqrt{t} y) & =2\left\langle\nabla_{y} X, \nabla_{y} Y\right\rangle+\left\langle\nabla_{\nabla_{y} y} X, Y\right\rangle \\
& =2\left\langle J_{X} y, \nabla_{y} Y\right\rangle-\left\langle\nabla_{Y} X, \nabla_{y} y\right\rangle=2\left\langle J_{X} y, \nabla_{y} Y\right\rangle .
\end{aligned}
$$

The integral (1.35) is then modified into

$$
\int_{M^{X}} \int_{N} \exp \left\{J_{X}-\frac{\left\langle(R(\cdot, \cdot)+i \nabla \cdot Y) y, J_{X} y\right\rangle}{2}-\frac{\left|J_{X} y\right|^{2}}{2}\right\} \mu .
$$

Proceeding as in (1.22), (1.23), the proof is finished.

\section{d) Integration Along the Fiber and Localization}

$B$ is another compact connected oriented Riemannian manifold. $Y$ is a Killing vector field over $B$. Let $B^{Y}$ be the submanifold of $B$

$$
B^{Y}=\{y \in B ; Y(y)=0\} .
$$

$f$ denotes a submersion of $M$ onto $B$, which is such that $f_{*} X=Y$. For $y \in Y$, set

$$
C_{y}=f^{-1}\{y\} \text {. }
$$

For every $y \in B, C_{y}$ is a submanifold of constant dimension. $y \rightarrow C_{y}$ defines a locally trivial fibration of $M$. 
Note that since $M$ and $B$ are oriented, $T C$ is an oriented bundle over $M$. Also the fibration $C$ is $X$ invariant, i.e. $e^{s X}$ sends a fiber $C_{y}$ in the fiber $C_{e^{s Y} y}$.

$T C$ is a subbundle of $T M$. Let $T^{H} M$ be the orthogonal bundle of $T C$ in $T M$. Then for every $x \in M, f_{*}$ is an isomorphism from $T_{x}^{H} M$ onto $T_{f(x)} B$.

Note that it we lift in $T_{X}^{H} M$ the scalar product of $T_{f(x)} B$ (while leaving the scalar product of $T C$ and the orthogonality of $T^{H} M$ and $T C$ unchanged), it is easy to verify that $X$ is still a Killing vector field for this new metric, essentially because $C$ is $X$ invariant, so that $T^{H} M$ is also $X$ invariant.

So from now on, we assume that $T^{H} M$ inherits the metric of $T B$.

Definition 1.5. $M^{Y}$ denotes the submanifold $f^{-1}\left(B^{Y}\right)$ of $M$.

For $y \in Y$, we define

$$
G_{y}=C_{y} \cap M^{X} .
$$

Note that for $x \in M^{Y}, f_{*} X(x)=0$, so that $X \in T C . X$ is then a Killing vector field over $M^{Y}$, and also if $y \in B^{Y}, X$ is a Killing vector field over $C_{y}$. It follows that if $y \in B^{Y}, G_{y}$ is a submanifold of $C_{y}$.

We now briefly describe $M^{X}$ in directions transverse to $C$.

Theorem 1.6. Take $x \in M^{X}$. Then $T_{x}^{H} M \cap T_{x} M^{Y} \subset T_{x} M^{X}$. For each $y \in B^{Y}, M^{X}$ is transversal to $C_{y}$ and intersects $C_{y}$ orthogonally. In particular, $y \in B^{Y} \rightarrow G_{y}$ is a (finite union of) locally trivial fibrations of $M^{X}$.

Proof. Take $x \in M^{X}$. Let $J_{X}^{\prime}$ be the infinitesimal action of $X$ in $T_{x} M^{Y} . J_{X}^{\prime}$ is an antisymmetric element of End $\left(T_{x} M^{Y}\right)$. Since the fiber $C_{f(x)}$ is $X$ invariant, $T_{x} C$ is invariant under $J_{X}^{\prime}$. Its orthogonal in $T_{x} M^{Y} T_{x}^{H} M \cap T_{x} M^{Y}$ is then also invariant under $J_{X}^{\prime}$.

Now for $s \in R, e^{s J_{X}^{\prime}}$ (which acts on $T_{x} M^{Y}$ ) coincides with $\left(e^{s X}\right)_{*}$. If $J_{X}^{\prime}$ was nonzero on $T_{x}^{H} M \cap T_{x} M^{Y}$, we could find $Z \in T_{x}^{H} M \cap T_{x} M^{Y}, Z \neq 0$, and $s$ such that $e^{s J_{X}^{\prime}} Z=-Z$, i.e. $\left(e^{s X}\right)_{*} Z=-Z$, which implies $f_{*}\left(e^{s X}\right)_{*} Z=-f_{*} Z$, i.e.

$$
\left(e^{s Y}\right)_{*} f_{*} Z=-f_{*} Z .
$$

Since $Z \in T_{x}^{H} M, f_{*} Z \neq 0$. Moreover, $f_{*} Z \in T_{f(x)} B^{Y}$. Since on $B^{Y}, e^{s Y}$ is the identity, $\left(e^{s Y}\right)_{*}$ is also the identity on $T_{f(x)} B^{Y}$. This contradicts $(1.50) . J_{X}^{\prime}$ is then equal to 0 on $T_{x}^{H} M \cap T_{x} M^{Y}$.

Now $T_{x} M^{X}$ is equal to $\operatorname{Ker} J_{X}^{\prime}$. It follows that $T_{x}^{H} M \cap T_{x} M^{Y} \subset T_{x} M^{X}$. Then $T_{x} C+T_{x} M^{X}=T_{x} M^{Y}$, so that $M^{X}$ is transversal to $C$. Also if $Z \in T_{x} M^{X}$ is orthogonal to $T_{x} M^{X} \cap T_{x} C, Z \in \operatorname{Ker} J_{X}^{\prime}$ and is orthogonal to $\operatorname{Ker} J_{X}^{\prime} \cap T_{x} C$. Then necessarily, $Z$ is orthogonal to $T_{x} C$. This shows that $M^{X}$ intersects $C$ orthogonally.

Definition 1.7. $N^{X}$ (respectively $N^{Y}$ ) denotes the normal bundle of $M^{X}$ (respectively $\left.B^{Y}\right)$ in $M$ (respectively $B$ ). $J_{X}$ (respectively $J_{X}^{\prime}, J_{Y}$ ) denotes the infinitesimal action of $X$ (respectively $X, Y$ ) in $N^{X}$ (respectively $N^{X} \cap T C, N^{Y}$ ).

We can lift $N^{Y}$ through $f$ as a subbundle of $T^{H} M$. We still note $N^{Y}$ the lifted bundle. We also lift $J_{Y}$ in a similar way.

We now have the key result.

Theorem 1.8. $N^{X} \cap T C$ coincides with the normal bundle of $G$ in $C$, and with the normal bundle of $M^{X}$ in $M^{Y}$. Moreover, $N^{X}$ splits into

$$
N^{X}=\left(N^{X} \cap T C\right) \oplus N^{Y} .
$$


On $M^{X}, J_{X}$ coincides with $J_{X}^{\prime}$ on $N^{X} \cap T C$, and with $J_{Y}$ on $N^{Y}$.

Proof. Since $M^{X}$ intersects $C$ orthogonally, $N^{X} \cap T C$ coincides with the normal bundle of $G$ in $C$. For the same reason, $N^{X} \cap T C$ coincides with the normal bundle of $M^{X}$ in $M^{Y}$.

Also on $M^{Y}$, we have $T M=T M^{Y} \oplus N^{Y}$, and $T M^{Y}$ and $N^{Y}$ are orthogonal in $T M$. Moreover, $T M^{X} C T M^{Y}$. It follows that $Z \in N^{X}$ if and only if its projection on $T M^{Y}$ is orthogonal to $T M^{X}$. Equation (1.51) is now obvious.

Clearly, $J_{X}$ coincides with $J_{X}^{\prime}$ on $N^{X} \cap T C$. Since $N^{Y}$ is orthogonal to $N^{X} \cap T C$ in $N^{X}, N^{Y}$ is stable by $J_{X}$. Moreover, since $f_{*} X=Y$, on $M^{X}$, we find that

$$
f_{*} J_{X}=J_{Y} f_{*} \text {. }
$$

Since $N^{Y}$ is stable under $J_{X}$, we find from (1.52) that on $N^{Y}, J_{X}=J_{Y}$.

Let $\nabla^{L}$ be the Levi-Civita connection on the manifold $M^{Y}$. Let $R^{L}$ be the curvature tensor of $\nabla^{L}$. $M^{X}$ being totally geodesic in $M^{Y}$, the Levi-Civita connection $\nabla^{L}$ induces an orthogonal connection on the normal bundle of $M^{X}$ in $M^{Y}$, i.e. on $N^{X} \cap T C$.

Also $N^{X} \cap T C$ is naturally oriented by the two form $Z, Z^{\prime} \in N^{X} \cap T C$ $\rightarrow\left\langle Z, J_{X}^{\prime} Z^{\prime}\right\rangle$. Since $T C$ is oriented, it follows that the fibration $G$ is naturally oriented over $B^{Y}$. If $Z, Z^{\prime} \in T M^{Y}, J_{X}^{\prime}+R^{L}\left(Z, Z^{\prime}\right)$ is an antisymmetric element of End $\left(N^{X} \cap T C\right)$, whose Pfaffian $\operatorname{Pf}\left[J_{X}^{\prime}+R^{L}\left(Z, Z^{\prime}\right)\right]$ is well defined.

Also by Bott and Tu [17, Chap. I], the integral along the fiber $C$ (or $G$ ) of any $C^{\infty}$ differential form is well-defined and produces a $C^{\infty}$ differential form on the base. We note $\int_{C}$ or $\int_{G}$ the integral along the fiber operators.

Also, if $v, v^{\prime} \in \Lambda\left(B^{Y}\right)$, we write

$$
v \equiv v^{\prime}
$$

if $v-v^{\prime}$ is exact. Finally, let $i$ be the embedding $M^{X} \underset{i}{\rightarrow} M, j$ the embedding $B^{Y} \underset{j}{\rightarrow} B$.

We now have the key result.

Theorem 1.9. Take $\mu \in \Lambda(M)$ which is $X$ equivariantly closed. Then

$$
j^{*} \int_{C} \mu \equiv \int_{G} \frac{i^{*} \mu}{\operatorname{Pf}\left[\frac{J_{X}^{\prime}+R^{L}}{2 \pi}\right]} .
$$

Proof. We give two proofs.

Proof No. 1. This proof is very similar to the Proof No. 2 of Theorem 1.3. To prove (1.54), we may as well assume that $B=B^{Y}$, i.e. that $Y=0$, so that $M=M^{Y}$.

$X \in T M$ can be identified with a 1 -form over $M^{Y}$, which we still note $X$. We claim that for any $s \in R$

$$
\int_{C} \mu \equiv \int_{C} \exp \left\{-s\left(d+i_{X}\right) X\right\} \mu .
$$

In fact, as in (1.11), we have

$$
\frac{\partial}{\partial s} \int_{C} \exp \left\{-s\left(d+i_{X}\right) X\right\} \mu=-\int_{C}\left(d+i_{X}\right)\left[\exp \left\{-s\left(d+i_{X}\right) X\right\} \mu\right] .
$$


Now by Bott and $\mathrm{Tu}\left[12\right.$, Chap. I], $\int_{C}$ and $d$ commute. Also since $X \in T C$, $\int_{C} i_{X} \ldots=0$. So we find from (1.56) that

$$
\frac{\partial}{\partial s} \int_{C} \exp \left\{-s\left(d+i_{X}\right) X\right\} \mu=-d\left[\int_{C} \exp \left\{-s\left(d+i_{X}\right) X\right\} \mu\right],
$$

and so (1.55) has been proved.

For any $t>0$, we get

$$
\int_{C} \mu \equiv \int_{C} \exp \left\{-\frac{\left(d+i_{X}\right) X}{2 t}\right\} \mu
$$

The proof now proceeds exactly as the Proof No. 2 of Theorem 1.3. In particular, we use (1.31), (1.34) and the fact that by Theorem 1.8, the infinitesimal action $J_{X}$ of $X$ on $T M$ coincides with $J_{X}^{\prime}$ on $N^{X} \cap T C$ and is 0 on the orthogonal of $N^{X} \cap T C$ and also that $J_{X}^{\prime}$ is non-degenerate on $N^{X} \cap T C$. The theorem is proved.

Proof No. 2. Take $v \in \Lambda\left(B^{Y}\right)$, which is $Y$-equivariantly closed. $j^{*} v$ is then closed on $B^{Y}$. Also $f^{*} v$ is $X$-equivariantly closed on $M$.

Using Theorem 1.3, we know that

$$
\int_{M}\left(f^{*} v\right) \mu=\int_{M^{X}} \frac{\left(f^{*} v\right) \mu}{\operatorname{Pf}\left[\frac{J_{X}+R}{2 \pi}\right]}=\int_{B^{Y}}\left(j^{*} v\right) \int_{G} \frac{i^{*} \mu}{\operatorname{Pf}\left[\frac{J_{X}+R}{2 \pi}\right]} .
$$

Also

$$
\int_{M}\left(f^{*} v\right) \mu=\int_{B} v \int_{C} \mu
$$

Since $f_{*} Y=X$, we have

$$
\left(d+i_{Y}\right) \int_{C} \mu=\int_{C}\left(d+i_{X}\right) \mu=0 .
$$

$v \int_{C} \mu$ is $Y$ equivariantly closed. Using Theorem 1.3 again, we find that if $R_{B}$ is the Levi-Civita curvature tensor of $B$,

$$
\int_{B} v \int_{C} \mu=\int_{B^{Y}} \frac{v \int_{C} \mu}{\operatorname{Pf}\left[\frac{J_{Y}+R_{B}}{2 \pi}\right]} .
$$

By a result of Witten [26] (also see Atiyah and Bott [4]), we know that $v \rightarrow j^{*} v$ surjects from $Y$ equivariantly closed forms on $B$ on the closed forms in $B^{Y}$. Using Poincaré duality on $B^{Y}$, we get from (1.59)-(1.62),

$$
\int_{G} \frac{i^{*} \mu}{\operatorname{Pf}\left[\frac{J_{X}+R}{2 \pi}\right]} \equiv \frac{j^{*} \int_{C} \mu}{\operatorname{Pf}\left[\frac{J_{Y}+R_{B}}{2 \pi}\right]} .
$$


Now by Theorem 1.8, $N^{X}$ splits according to (1.51). Also we know that $1 / \mathrm{Pf}\left[\frac{J_{X}+R}{2 \pi}\right]$, as a standard characteristic class over $M^{X}$, defines a closed form over $M^{X}$ whose cohomology class is independent of any Euclidean connection on $N^{X}$ preserving $J_{X}$. Still using Theorem 1.8, we get

$$
\frac{1}{\operatorname{Pf}\left[\frac{J_{X}+R}{2 \pi}\right]} \equiv \frac{1}{\operatorname{Pf}\left[\frac{J_{Y}+R_{B}}{2 \pi}\right] \operatorname{Pf}\left[\frac{J_{X}^{\prime}+R^{L}}{2 \pi}\right]} .
$$

Using (1.63) and (1.64), we finally obtain (1.54).

Remark 3. The reader will have noticed that also in Proof No. 2, we could have directly assumed that $Y=0, M^{Y}=M$, so that (1.54) would have been proved immediately by a proof which would be in fact equivalent to Proof No. 1 . However, our purpose is to produce a proof which can eventually be generalized in infinite dimensions, for which Proofs No. 1 and 2 seem to be quite different. It turns out that Proof No. 2 is exactly what is needed to understand our second proof in [15] of the Index Theorem for families.

Note that as a consequence of Bismut [13, Theorem 3.9], the result of Witten [26] mentioned in the proof extends on the loop space, at least for even forms, essentially because

$$
K(B) \otimes_{Z} R \sim H^{* \operatorname{even}}(B, R) .
$$

Not unexpectedly, although the result of Witten [26] is not needed in our second proof of the Index Theorem for families in [15], (1.65) is needed explicitly!

Also note that $R^{L}$ appears explicitly in Proof No.1. This is of utmost importance in understanding our first proof [15] of the Index Theorem for families. We refer in particular to Theorems 3.15 and 3.18 , where (1.34) is proved in an infinite dimensional situation.

\section{The Probabilistic Proof of the Index Theorem}

In this section, we will essentially show that the probabilistic proof of the AtiyahSinger Index Theorem for Dirac operators is by itself the rigorous infinite dimensional version of Proof No. 2 of the localization formula in equivariant cohomology given in Theorem 1.3. This shows that the remark of Atiyah and Witten [2], who showed that, formally the Index of the Dirac operators on the spin complex could be computed using formula (1.3) can be much extended, in the sense that the heat equation method produces by itself the corresponding rigorous proof, the finite dimensional and infinite dimensional proofs being strictly parallel.

In a), we give the main assumptions and notations. In b), we recall some elementary facts on the geometry of the loop space of a Riemannian manifold. In c), the Dirac operator is described. In d), we briefly recall the results in Atiyah [2] and ourselves [13] on the relations of Index Theory to the cohomology of the loop space. In e), we describe our probabilistic proof of the Index Theorem [12] and compare it with Proof No. 2 of Theorem 1.3. In f), we describe our heat equation proof [14] of the infinitesimal Lefschetz formulas, and compare it with the proof of Theorem 1.4 . 


\section{a) Assumptions and Notations}

$M^{0}$ now denotes a compact connected Riemannian manifold of even dimension $n=2 l . N^{0}$ is the $\mathrm{SO}(n)$ principal bundle of oriented orthonormal frames in $T M^{0}$. For $x \in M^{0}$, the fiber $N_{x}^{0}$ is the set of oriented linear isometries from the canonical oriented Euclidean space $R^{n}$ into $T_{x} M^{0}$.

We assume that $M^{0}$ is a spin manifold, i.e. the $\mathrm{SO}(n)$ principal bundle $N^{0}$ lifts to a Spin $(n)$ principal bundle $N^{\prime 0}$ so that the projection $N^{\prime 0} \rightarrow N^{0}$ induces the covering mapping $\operatorname{Spin}(n) \underset{\sigma}{\rightarrow} \mathrm{SO}(n)$ on each fiber.

Let $S$ be the $2^{l}$ dimensional Hermitian Space of spinors which splits into $S=S_{+} \oplus S_{-}$, where $S_{+}, S_{-}$are the $2^{l-1}$ dimensional spaces of positive and negative spinors. $S_{+}, S_{-}$are orthogonal in $S$.

Let $F, F_{+}, F_{-}$be the bundles of spinors over $M^{0}$.

$$
F=N^{\prime 0} \times_{\operatorname{Spin}(n)} S, \quad F_{ \pm}=N^{\prime 0} \times \times_{\operatorname{Spin}(n)} S_{ \pm} .
$$

On $N^{0}$, we put the Levi-Civita connection, which lifts naturally to $N^{\prime 0}$. Let $R$ be the curvature tensor of $T M$. Also let $\xi$ be a $k$-dimensional Hermitian bundle over $M$, endowed with a unitary connection, whose connection form is $\lambda$ and curvature form is $\Lambda$. $\Lambda$ is the equivariant representation of the curvature tensor $L$. Let $\pi$ be the projection $\xi \rightarrow M^{0}$. $\nabla$. denotes the covariant differentiation operator for any of the considered connections.

Recall that $T M$ acts on $F$ by Clifford multiplication, and exchanges $F_{+}$and $F_{-}$. Also if $e \in T M, e$ act on $F \otimes \xi$ by the Clifford multiplication $e$ on $F$, i.e. by $e \otimes 1$. In the sequel, we will write $e$ instead of $e \otimes 1$.

If $G$ is any fiber bundle over $M^{0}, \Gamma(G)$ is the set of $C^{\infty}$ section of $G, e_{1}, \ldots, e_{n}$ is an orthonormal base of $T^{M}$.

Definition 2.1. D denotes the Dirac operator acting on $\Gamma(F \otimes \xi)$,

$$
D=\sum_{1}^{n} e_{i} \nabla_{e_{i}}
$$

$D$ interchanges $\Gamma\left(F_{+} \otimes \xi\right)$ and $\Gamma\left(F_{-} \otimes \xi\right)$. Let $D_{+}, D_{-}$be the restrictions of $D$ to $\Gamma\left(F_{+} \otimes \xi\right), \Gamma\left(F_{-} \otimes \xi\right)$.

When acting on $\Gamma\left(F_{+} \otimes \xi\right) \oplus \Gamma\left(F_{-} \otimes \xi\right)$, we may write $D$ in matrix form as

$$
D=\left[\begin{array}{cc}
0 & D_{-} \\
D_{+} & 0
\end{array}\right] \text {. }
$$

$D$ is formally self-adjoint.

\section{b) The Loop Space and the Action of $S_{1}$}

$M$ denotes the set of $C^{\infty}$ mappings from $S_{1}=R / Z$ into $M^{0}$. If $x . \in M$, the tangent space $T_{x} M$ is identified with the set of smooth periodic vector fields $X$. over $x$., such that $X_{s} \in T_{x_{s}} M^{0}$. If $Y, Z \in T_{x} M$, we define the scalar product

$$
\langle Y, Z\rangle=\int_{0}^{1}\left\langle Y_{s}, Z_{s}\right\rangle d s .
$$

$M$ is then a Riemannian infinite dimensional manifold. 
We now follow Atiyah [2]. Namely, we note that $S_{1}$ acts on $M$ by the mappings $k_{s}$ defined by $k_{s} x_{.}=x_{s+.} \cdot k_{s}\left(s \in S_{1}\right)$ is a group of isometries, whose generating Killing vector field $X$ is given by

$$
X(x)_{s}=\left(\frac{d x}{d s}\right)_{s} .
$$

Clearly, $M^{0}$ coincides with the set $M^{X}$ of the zeros of $X$.

Also the Levi-Civita connection of $M^{0}$ extends in an obvious way as the LeviCivita connection on $M$. In fact, if $X, Y \in T M$, if for $x \in M$, we set

$$
\left[\left(\nabla_{X} Y\right)_{x}\right]_{s}=\nabla_{X_{s}} Y_{s}
$$

Equation (2.6) defines the Levi-Civita connection of $M$.

We now have

Theorem 2.2. Take $x \in M, Y \in T M$. If $\frac{D}{D S}$ is the covariant differentiation operator along $s \rightarrow x_{s}$, then

$$
\nabla_{Y} X=\frac{D}{D S} Y_{s}, \quad \nabla_{Y}(\nabla \cdot X)+R(X, Y)=0 .
$$

If $X$ is identified with the 1 -form $Y \rightarrow \int_{0}^{1}\langle X, Y\rangle d$ s, then for $Y, Z \in T_{x} M$

$$
d X(Y, Z)=2 \int_{0}^{1}\left\langle\frac{D Y}{D s}, Z\right\rangle d s
$$

Proof. We can always assume that $t \in R \rightarrow x^{t} \in M$ is $C^{\infty}$ and also that $\frac{\partial x_{s}^{t}}{\partial t}=Y_{s}$. Then $[X, Y]=0$, and so since $\nabla$ does not have torsion

$$
\left(\nabla_{Y} X\right)_{s}=\left(\nabla_{X} Y\right)_{s}=\frac{D}{D S} Y_{s} .
$$

Also for $Y, Z \in T M$,

$$
\nabla_{Y}(\nabla \cdot X) Z=\nabla_{Y} \nabla_{Z} X-\nabla_{\nabla_{Y} Z} X=\nabla_{Y} \frac{D}{D S} Z-\frac{D}{D S} \nabla_{Y} Z=R(Y, X) Z .
$$

Equation (2.7) is proved, (2.8) is left to the reader.

Remark 1 . Since $X$ is a Killing vector field, the second equation in (2.7) is also a consequence of (1.27).

c) The Index of the Dirac Operator

Recall that the index of $D_{+}$is defined by

$$
\text { Ind } D_{+}=\operatorname{dim} \operatorname{Ker} D_{+}-\operatorname{dim} \operatorname{Ker} D_{-} .
$$

Consider the splitting

$$
F \otimes \xi=\left(F_{+} \otimes \xi\right) \oplus\left(F_{-} \otimes \xi\right) .
$$


We may consider $F \otimes \xi$ as a $Z_{2}$ graded vector bundle, in which the even elements are in $F_{+} \otimes \xi$, and the odd ones in $F_{-} \otimes \xi$. Let $\tau$ be the involution defining the grading, i.e. if $e \in F_{+} \otimes \xi, \tau e=(-1)^{\operatorname{deg} e} e$.

Similarly,

$$
\Gamma(F \otimes \xi)=\Gamma\left(F_{+} \otimes \xi\right) \oplus \Gamma\left(F_{-} \otimes \xi\right) .
$$

We still note by $\tau$ the involution in (2.11) defining the $Z_{2}$ grading.

We also recall Lichnerowicz's formula [22] for $D^{2}$. Namely, let $\Delta^{I I}$ be the Bochner horizontal Laplacian on $\Gamma(F \otimes \xi)$. Then, if $K$ is the scalar curvature of $M$,

$$
D^{2}=-\Delta^{H}+\frac{K}{4}+\frac{1}{2} e_{i} e_{j} \otimes L\left(e_{i}, e_{j}\right) .
$$

For $t>0, e^{-\frac{t D^{2}}{2}}$ is a self-adjoint operator on $\Gamma\left(F_{ \pm} \otimes \xi\right)$. It is given by a kernel $P_{t}\left(x, x^{\prime}\right)$ which is $C^{\infty}$ in $\left(x, x^{\prime}\right)$.

By Atiyah et al. [5, Theorem E III], we know by a standard spectral theory argument that

$$
\operatorname{Ind} D_{+}=\operatorname{Tr}_{+} e^{-\frac{t D^{2}}{2}}-\operatorname{Tr}_{-} e^{-\frac{t D^{2}}{2}}
$$

where $\mathrm{Tr}_{+}, \mathrm{Tr}_{-}$are the traces of operators acting on $\Gamma\left(F_{+} \otimes \xi\right), \Gamma\left(F_{-} \otimes \xi\right)$.

We define the supertrace $\operatorname{Tr}_{s} A$ of a trace class operator $A$ acting on $\Gamma(F \otimes \xi)$ by

$$
\operatorname{Tr}_{s} A=\operatorname{Tr} \tau A .
$$

Then (2.13) writes

$$
\text { Ind } D_{+}=\operatorname{Tr}_{s} e^{-\frac{t D^{2}}{2}} \text {. }
$$

For $x \in M, P_{t}(x, x) \in \operatorname{End}(F \otimes \xi)_{x}$ and $P_{t}(x, x)$ sends $\left(F_{ \pm} \otimes \xi\right)_{x}$ into itself. Equation (2.13) is equivalent to

$$
\text { Ind } D_{+}=\int_{M} \operatorname{Tr}_{s}\left[P_{t}(x, x)\right] d x .
$$

\section{d) Equivariant Cohomology on the Loop Space and the Index Theorem}

We now recall the results of Atiyah [2] and our results [13]. Namely, we identify $X$ with a 1 -form over $M$ using the Riemannian metric. Let $\eta^{0}$ be the $U(k)$ principal bundle over $M^{0}$ of the unitary frames in $\xi$.

Let $\eta$ be the bundle over $M$ of the $C^{\infty}$ sections $s \in S_{1} \rightarrow v_{s} \in \eta^{0}$. There is a natural projection $\varrho \eta \rightarrow M$.

For $v \in \eta, \Lambda_{v_{s}}$ is identified with the 2 -form over $\eta$,

$$
Z, Z^{\prime} \in T_{v} \eta \rightarrow \Lambda_{v_{s}}\left(Z_{s}, Z_{s}^{\prime}\right) .
$$

$\Lambda(\eta)$ denotes the exterior algebra of $T^{*} \eta$.

We now follow Bismut [13, Definition 3.6].

Definition 2.3. For $v \in \eta, H_{s}^{\prime}$ denotes the element of End $C^{k} \otimes \Lambda_{v}^{\text {even }}(\eta)$ defined by the differential equation

$$
d H_{s}^{\prime}=H_{s}^{\prime}\left[\lambda\left[\frac{d v}{d s}\right]+\Lambda_{v_{s}}\right], \quad H^{\prime}(0)=I .
$$


We now recall the result in [13, Theorem 3.9].

Theorem 2.4. The even form over $\eta \operatorname{Tr} H_{1}^{\prime}$ projects on $M$ as an even form $\beta$. $\beta$ is $X$-equivariantly closed.

Remark 1. For an interpretation of (2.15) in terms of standard equivariant characteristic classes (in the sense of Berline and Vergne [9]) and Kac-Moody algebras, see [13, Remark 3.2].

We now recall the formal result which Atiyah [2] obtained for the Dirac operator acting for the spin complex, and which we extend in [13] to twisted spin complexes. Namely, it follows from Atiyah [2], and ourselves [13], that the rigorous formula (2.16) can be written formally as

$$
\operatorname{Ind} D_{+}=C \int_{M} \exp \left\{-\frac{\left(d+i_{X}\right) X}{2 t}\right\} \beta,
$$

where $C$ is the infinite normalizing constant.

$$
C=\frac{\left(\int_{1}^{+\infty} m^{2}\right)^{l}}{(2 \pi)^{l}} i^{l} .
$$

Of course, the Wiener measure is carried by the set of continuous paths (or loops) and gives 0 measure to $M$. For simplicity, we will forget about this, keeping in mind that all the standard operations on $C^{\infty}$ paths can be done in a measure theoretic sense on continuous paths.

We now do a few remarks on (2.18):

- The transformation of (2.13) into (2.18) is obtained using

a) Lichnerowicz's formula.

b) A highly non-trivial connection between the Trace of the $\operatorname{Spin}(n)$ representation and the renormalized Pfaffian of $\nabla . X$ on TM. Namely, Atiyah and Witten [2] noted that if $x$ is a smooth loop, if $\tau_{0}^{1}$ is the parallel transport operator from $F_{ \pm, x_{0}}$ into $F_{ \pm, x_{0}}$ along $s \rightarrow x_{1-s}$, if $\operatorname{Tr}_{ \pm} \tau_{0}^{1}$ is the corresponding trace, if $\operatorname{Pf}(-d X)$ is the formal Pfaffian of $-d X$, then

$$
\frac{i^{l} \operatorname{Pf}\left[-\frac{d X}{2}\right]}{\left(\prod_{1}^{\infty} 4 \pi^{2} m^{2}\right)^{l}}=\left(\operatorname{Tr}_{+}-\operatorname{Tr}_{-}\right)\left(\tau_{0}^{1}\right) .
$$

This transformation is crucial in connecting a concept of operator theory (which is the Index) into a cohomological expression, which is the right-hand side of (2.18).

Also note the following facts:

- Equation (2.18) is strictly similar to the right-hand side of (1.25).

- It is impossible to make $t=+\infty$ in (2.16). Remark 2 in Sect. 1 entirely applies here, since $\int_{M} \beta$ in general is not well defined.

\section{e) The Heat Equation as a Natural Proof of Localization in Infinite Dimensions}

As we shall see, although the right-hand side of (2.18) is formal, if we interpret correctly our heat equation proof of the Index Theorem, we find it reproduces step by step Proof No. 2 of Theorem 1.3. 
To simplify the discussion, we assume that $\xi$ is the trivial bundle. The general case can be treated using similar arguments. Let $E_{x_{0}, x_{0}}^{t}$ be the probability law of the Brownian bridge $x$. with $x(0)=x(1)=x_{0}$, associated with the scaled Laplacian $\frac{t \Delta}{2}$ (see [10, Chap. 2]). Let $\tau_{0}^{1, t}$ be the parallel transport operator from fibers over $x_{0}$ into themselves along $s \rightarrow x_{1-s}$.

By [12, Theorem 2.5], if $p_{t}(x, y)$ is the heat equation kernel associated with the operator $e^{\frac{t \Delta}{2}}$, we have

$$
\operatorname{Tr}_{s}\left\{P_{t}\left(x_{0}, x_{0}\right)\right\}=p_{t}\left(x_{0}, x_{0}\right) E_{x_{0}, x_{0}}^{t}\left[\exp \left[-t \int_{0}^{1} \frac{K\left(x_{s}\right)}{8} d s\right] \operatorname{Tr}_{s} \tau_{0}^{1, t}\right]
$$

To calculate (2.21), we use our results in [10, Sect. 4], which turn out to be strictly equivalent to what is done after (1.25). Namely, if $x_{0} \in M^{0}=M^{X}$, we identify the tangent bundle $T_{x_{0}} M^{0}$ to the set of constant mappings $s \in S_{1} \rightarrow v^{2} \in T_{x_{0}} M^{0}$, which we call also $H_{2}^{\prime}$. The normal bundle $N_{x_{0}}$ is the set $H_{1}^{\prime}$ of $L_{2}$ mappings $s \in S_{1}$ $\rightarrow w^{1} \in T_{x_{0}} M$ such that $\int_{0}^{1} w_{s}^{\prime 1} d s=0$.

For simplicity, we restrict ourselves to $C^{\infty}$ functions $w_{s}^{\prime 1}$. Note that if $H_{1}$ is the set of $C^{\infty}$ mappings $s \in S_{1} \rightarrow w_{s}^{1}$ such that $w_{0}^{1}=w_{1}^{1}=0, H_{1}^{\prime}$ and $H_{1}$ are in one-to-one correspondence by

$$
w_{1}^{\prime 1} \in H_{1}^{\prime} \rightarrow w^{1} \in H_{1}: w_{s}^{1}=w_{s}^{\prime 1}-w_{0}^{\prime 1} .
$$

Also the infinitesimal action $J_{X}$ of $X$ on $H_{1}^{\prime}$ is given by

$$
J_{X} w_{s}^{1}=\frac{d w_{s}^{\prime 1}}{d s} .
$$

We now explain how in [10, Chap. 4], [12, Sect. 2], we obtain the infinite dimensional analogue of the diffeomorphism $f_{U}$ described after (1.27).

Namely, take $w^{1} \in H_{1}^{\prime}$. Let $w^{1}$ be the corresponding element in $H_{1}$. For $v_{2} \in H_{2}$, consider the curve $s \rightarrow x_{s}$ in $M^{0}$ such that

- $x(0)=x_{0}$.

- The Cartan development of $s \rightarrow x_{s}$ in $T_{x_{0}} M^{0}$ coincides with $w_{s}^{1}+s v^{2}$.

This means that $\frac{d x_{s}}{d s}$ is the parallel transport of $\frac{d w_{s}^{1}}{d s}+v^{2}$ along $x_{u}(0 \leqq u \leqq s)$.

If $w$. is close enough to 0 (for an adequate norm), the implicit function Theorem shows that there is a unique $v^{2} \in H_{2}$ such that $x_{1}=x_{0}$.

If $M^{x_{0}}$ is the set of $x \in M$ such that $x(0)=x(1)=x_{0}$, we have then found a natural parametrization of a neighborhood of the constant loop $x_{0}$ by a neighborhood of $\left(x_{0}, 0\right)$ in $H_{1}^{\prime}:\left(x_{0}, w^{\prime 1}\right) \rightarrow x .=f\left(x_{0}, w^{\prime 1}\right)$.

This is exactly what is done in $[10,12]$, with the difficulty that $w^{1}, w^{1}, x$ are not smooth, that everything has to be done in a measure theoretic sense etc. .... The change of variables $y=\sqrt{t} y^{\prime}$ described before (1.28) corresponds exactly to scaling $w^{\prime 1}$ by a factor $\sqrt{t}$. In [10, Theorem 4.16 and its corollary] and in [12, Theorem 2.15], we then find that if $H_{1}$ is endowed with the flat Brownian bridge measure 
(which gives 0 measure to $C^{\infty}$ paths ...), as $t \downarrow \downarrow 0$, for any $k \in N$

$$
\operatorname{Tr}_{s}\left[P_{t}\left(x_{0}, x_{0}\right)\right]=\int \frac{\left[\operatorname{Tr}_{s} \tau_{0}^{1, t}\right]}{(\sqrt{2 \pi t})^{n}} H\left(\sqrt{t} d w^{1}\right) d P_{1}\left(w^{1}\right)+o\left(t^{k}\right),
$$

where as $t \downarrow \downarrow 0$

$$
H\left(\sqrt{t} d w^{1}\right) \rightarrow 1, \quad \text { boundedly . }
$$

Also if $\tau_{0}^{1, t}$ is considered as an element of $\mathrm{SO}(n)$ acting on $T_{x_{0}} M$, it is trivial to verify that

$$
\tau_{0}^{1, t}=I-\frac{t \int_{0}^{1} R_{x_{0}}}{2}\left(d w_{s}^{1}, w_{s}^{1}\right)+o(t) .
$$

Note that we can as well replace $w^{1}$ by $w^{\prime 1}$ in (2.21), since adding constants has no effect on $\int_{0}^{1} R_{x_{0}}\left(d w_{s}^{1}, w_{s}^{1}\right)$.

An argument from representation theory [12, Theorem 1.5] shows that

$$
\lim _{t \downarrow \downarrow 0} \frac{\operatorname{Tr}_{s} \tau_{0}^{1, t}}{t^{n / 2}}=(i)^{l} \mathrm{Pf}\left[-\int_{0}^{1} \frac{R_{x_{0}}\left(d w_{s}^{1}, w_{s}^{1}\right)}{2}\right] .
$$

Recalling that if $A$ is an antisymmetric $(n, n)$ matrix

$$
(\operatorname{Pf} A) d x^{1} \wedge \ldots \wedge d x^{n}=\frac{A^{\wedge l}}{l !}
$$

we find from $(2.16),(2.23),(2.26)$ that if $\int_{0}^{1} R_{x_{0}}\left(d w^{1}, w^{1}\right)$ is identified with the 2-form $(Y, Z) \rightarrow\left\langle Y, \int_{0}^{1} R_{x_{0}}\left(d w^{1}, w^{1}\right) Z\right\rangle$, then

$$
\operatorname{Ind} D_{+}=\frac{i^{l}}{(2 \pi)^{l}} \int_{M^{0}} \int\left[\exp \left\{-\int_{0}^{1} \frac{R\left(d w^{1}, w^{1}\right)}{2}\right\}\right] d P_{1}\left(w^{1}\right) .
$$

We now use the well-known symmetries of the Levi-Civita curvature tensor $R$ to get

$$
\int_{0}^{1}\left\langle Y, R\left(d w^{1}, w^{1}\right) Z\right\rangle=\int_{0}^{1}\left\langle R(Y, Z) w^{1}, d w^{1}\right\rangle
$$

which is a key step in the proof, which corresponds exactly to (1.34).

Using (2.28), we find that

$$
\text { Ind } D_{+}=\frac{i^{l}}{(2 \pi)^{l}} \int_{M^{0}} \int \exp \left\{-\frac{\int_{0}^{1}\left\langle R(\cdot, \cdot) w^{1}, d w^{1}\right\rangle}{2}\right\} d P_{1}\left(w^{1}\right) .
$$

Using formula (2.22) for $J_{X}$ and the fact that at least formally, if $\mathscr{D}\left(w^{1}\right)$ is the "Lebesgue measure" on $H_{1}$,

$$
d P_{1}\left(w^{1}\right)=C \exp \left\{-\int_{0}^{1}\left|\frac{d w^{1}}{d s}\right|^{2} d s\right\} \mathscr{D}\left(w^{1}\right),
$$

we find that (2.30) is identical to (1.35). 
More generally, if $\xi$ is any unitary bundle instead of (2.30), we get

$$
\text { Ind } D_{+}=\frac{(i)^{l}}{(2 \pi)^{l}} \int_{M^{0}} \int \exp \left\{-\frac{\int_{0}^{1}\left\langle R(\cdot, \cdot) w^{1}, d w^{1}\right\rangle}{2}\right\} d P_{1}\left(w^{1}\right) \beta \text {. }
$$

The reasoning of $[12$, Sect. 3$]$ is still very close to what has been done before.

This shows that in its very details, if we start from (2.13), the proof of (2.30), (2.31) is exactly identical to Proof No. 2 of Theorem 1.3.

In [12], the final evaluation of (2.30), (2.31) is done using a formula of P. Lévy, which as is well-known, it is equivalent to the computation of an infinite determinant corresponding to $\operatorname{Pf}\left[\frac{J_{X}+R}{2 \pi}\right]$. So we find the Index formula of Atiyah-Singer

$$
\text { Ind } D_{+}=\int_{M^{0}} \hat{A}\left(\frac{R}{2 \pi}\right) \operatorname{ch} \xi .
$$

Remark 2. Consider any connection on $T M^{0}$ which preserves the metric of $T M^{0}$ but whose torsion $T$ is non 0 . Let $D^{\prime}$ be the Dirac operator calculated with this new connection. $D_{+}^{\prime}$ has the same index as $D_{+}$. Also, even if $D^{\prime}$ is in general not selfadjoint, the formula (2.15) still holds with $D_{+}$replaced by $D_{+}^{\prime}$. This fact [which is a consequence of the formalism of Quillen [25] described in Sect. 3a)] is briefly proved in [15, Theorem 5.3]. However, the heat equation method on $D^{\prime}$ does not converge in general.

The interpretation for this is very simple. Namely, let $\langle T \wedge \theta\rangle$ denote the antisymmetrization of the $(3,0)$ tensor, $(X, Y, Z) \rightarrow\langle T(X, Y), Z\rangle .\langle T$ 今 $\theta\rangle$ obviously extends as a $(3,0)$ tensor on $M$ by setting

$$
\langle T \wedge \theta\rangle(X, Y, Z)=\int_{0}^{1}\langle T \wedge \theta\rangle\left(X_{s}, Y_{s}, Z_{s}\right) d s .
$$

$\langle T, \theta\rangle$ is clearly $X$ invariant. Now by proceeding as in Atiyah [2] and Bismut [13], it is not difficult to find that the rigorous trace formula for the index of $D_{+}^{\prime}$ can be written formally as

$$
\operatorname{Ind} D_{+}^{\prime}=C \int_{M} \exp \left\{-\frac{\left(d+i_{X}\right) X}{2 t}\right\} \exp \left\{-\frac{\left(d+i_{X}\right)\langle T \wedge \hat{\wedge} \theta\rangle}{2 \mathrm{t}}\right\} \beta \text {. }
$$

Equation (2.33) shows in an obvious cohomological way that Ind $D_{+}^{\prime}$ does not depend on the connection. It has also the merit of exhibiting in the most obvious way why, in general, localization will not take place as $t \downarrow \downarrow 0$. A similar formula also appears in (3.58) in connection with the Index Theorem for families. Equation (2.33) can be extended to the case of more general perturbations of $D$.

\section{f) The Infinitesimal Lefschetz Formulas}

Let $Y$ be a Killing vector field on $M^{0}$. $Y$ obviously extends as a Killing vector field on $M$ by setting for $x_{s} \in M,[Y(x .)]_{s}=Y\left(x_{s}\right)$. It is then obvious that $[X, Y]=0$. To simplify the discussion, we assume that $\xi$ is the trivial line bundle. 
$Y$ acts naturally by the Lie derivative operator $L_{Y}$ on $T M^{0} . L_{Y}$ has a natural lift to $\Gamma\left(F_{ \pm}\right) \cdot e^{Y}$ then acts on $\operatorname{Ker} D_{+}$and $\operatorname{Ker} D_{-}$. By definition, the Lefschetz number of $e^{Y}$ is given by

$$
L\left(e^{Y}\right)=\operatorname{Tr}_{\mathrm{Ker}^{D_{+}}} e^{Y}-\operatorname{Tr}_{\mathrm{Ker} D_{-}} e^{Y} .
$$

By a well-known formula, which is obvious using spectral theory, we know that for any $t>0$,

$$
L\left(e^{Y}\right)=\operatorname{Tr}_{s} e^{L_{Y}-\frac{t D^{2}}{2}} .
$$

In [13, Sect. 3d)], we have shown how formally we can write

$$
L\left(e^{Y}\right)=C \int_{M} \exp \left\{-\frac{\left(d+i_{X+Y}\right)(X+Y)}{2 t}\right\},
$$

where $C$ is given by (2.19). The proof of the Lefschetz fixed points formulas of Atiyah and Bott [3] and Atiyah and Singer [6] is obtained by making $t \downarrow \downarrow 0$ in (2.35)(see [12, Sect. 4]). The proof of [12] is formally much connected with Proof No. 2 of Theorem 1.3 .

We now concentrate on the infinitesimal Lefschetz formulas. In [14, Theorem 1.6] we proved that for any $z \in C, t>0$, if $Y$ acts by Clifford multiplication on $\Gamma(F \otimes \xi)$, then

$$
-\frac{t}{2}\left(D+\frac{z Y}{2 t}\right)^{2}-z L_{Y}=\frac{t}{2} \sum_{1}\left(\nabla_{e_{2}}-\frac{z\left\langle Y, e_{i}\right\rangle}{2 t}\right)^{2}-\frac{t K}{8} .
$$

Let $S_{t}^{z}\left(x, x^{\prime}\right)$ be the $C^{\infty}$ kernel associated with the operator $e^{-\frac{t}{2}\left(D+\frac{z Y}{2 t}\right)^{2}-z L_{Y}}$.

In [14, Theorem 1.9], we proved that

$$
L\left(e^{z Y}\right)=\int_{M} \operatorname{Tr}_{s}\left[S_{t}^{z}(x, x)\right] d x .
$$

Using (2.37), and the notations of (2.21), we proved in [14, Theorem 2.2],

$$
\begin{aligned}
\operatorname{Tr}_{s}\left[S_{t}^{z}\left(x_{0}, x_{0}\right)\right]= & p_{t}\left(x_{0}, x_{0}\right) E_{x_{0}, x_{0}}^{t} \\
& {\left[\exp \left\{-t \int_{0}^{1} \frac{K\left(x_{s}\right) d s}{8}-\frac{z}{2 t} \int_{0}^{1}\left\langle Y\left(x_{s}\right), d x_{s}\right\rangle\right\} \operatorname{Tr}_{s} \tau_{0}^{1, t}\right] . }
\end{aligned}
$$

By proceeding as Atiyah [2], we obtain from (2.39) the formal representation

$$
L\left(e^{z Y}\right)=C \int_{M} \exp \left\{-\frac{\left(d+i_{X+{ }_{z} Y}\right) X}{2 t}\right\} .
$$

The right-hand side of (2.40) is strictly identical to the right-hand side of (1.42).

Also we proved in [14, Theorem 2.9] that as $t \downarrow \downarrow 0,(2.39)$ has a limit. In comparison with the proof of the Index Theorem ([12] and Sect. 2e)), the key fact is that using the notations of Sect. 2e), if $x_{s}^{t}$ is associated with $\sqrt{t} w^{1}$,

$$
\lim _{t \downarrow \downarrow 0} \int_{0}^{1} \frac{\left\langle Y\left(x_{s}^{t}\right), d x_{s}^{t}\right\rangle}{t}=\int_{0}^{1}\left\langle\nabla_{w_{s}^{1}} Y\left(x_{0}\right), d w_{s}^{1}\right\rangle .
$$


If $z \in i R$,

$$
\left|\exp \left\{-\frac{z \int_{0}^{1}\left\langle Y\left(x_{s}^{t}\right), d x_{s}^{t}\right\rangle}{2 t}\right\}\right|
$$

is bounded.

So we prove rigorously in $[14$, Theorem 2.10$]$ that if $z \in i R$,

$$
L\left(e^{z Y}\right)=\frac{i^{l}}{(2 \pi)^{l}} \int_{M^{0}} \int \exp \left\{-\int_{0}^{1} \frac{\left\langle(R(\cdot, \cdot)+z \nabla \cdot Y) w^{1}, d w^{1}\right\rangle}{2}\right\} d P_{1}\left(w^{1}\right) .
$$

Equation (2.43) is then computed using a formula of P. Lévy.

Note that the proof of [14], which has been briefly sketched here, is very close to the proof of Theorem 1.4.

We now briefly explain how we guessed (2.37). We wrote formally from (2.36)

$$
L\left(e^{z Y}\right)=C \int_{M} \exp \left\{-\frac{\left(d+i_{X+z Y}\right) X}{2 t}\right\} \exp \left\{-\frac{\left(d+i_{X+z Y}\right) Y}{2 t}\right\} .
$$

Now as a 1 -form, $Y$ is $X+z Y$ invariant so that

$$
\exp \left\{-\frac{\left(d+i_{X+z Y}\right) Y}{2 t}\right\}-1
$$

is $X+z Y$ exact. Our conjecture was that although $M$ is non-compact, in (2.44), we can replace $\exp \left\{-\frac{\left(d+i_{X+z Y}\right) Y}{2 t}\right\}$ by 1 . This is how we were led to prove (2.37) and (2.39).

\section{Integration Along the Fiber in the Loop Space, and the Index Theorem for Families}

We now will show how our proofs in [15] of the Index Theorem for families are related to the proofs of Theorem 1.9. In a), we briefly explain the construction by Quillen [25] of the Chern character of a finite dimensional difference bundle using superconnections. In b), we briefly describe a fiber manifold $M^{0} \underset{x}{\rightarrow} B^{0}$. In c), we introduce connections on infinite dimensional bundles over the base $B^{0}$. In d), we construct the Dirac operator $D$ along the fibers. In e), we use Quillen's formalism in an infinite dimensional context, to describe the infinite dimensional formula for the Chern character of $\operatorname{Ker} D-\operatorname{Coker} D$ given in [15]. In f), the Levi-Civita superconnection is introduced. In g), the first proof of [15] of the Index Theorem for families is briefly sketched. In h), the second proof [15] is described. Finally, in i), we show how at least formally, the formalism of superbundles, superconnections can be seen as an operator theoretic description of the integration along the fiber in the loop space. 


\section{a) The Construction by Quillen of the Chern Character of a Difference Bundle}

We here follow Quillen [25]. Let $N$ be a compact connected manifold. $E=E_{0} \oplus E_{1}$ is a $Z_{2}$ graded (or super) vector bundle, $\tau$ is the involution defining the grading: $\tau e=(-1)^{\mathrm{deg} e} e$.

Then End $E$ is a $Z_{2}$ graded algebra, the even (odd) elements commuting (respectively anticommuting) with $\tau$. $[x, y]_{s}$ denotes the supercommutator of $x, y \in$ End $E$

$$
[x, y]_{s}=x y-(-1)^{\operatorname{deg} x \operatorname{deg} y} y x .
$$

$\operatorname{Tr}_{s} x$ denotes the supertrace of $x$, i.e.

$$
\operatorname{Tr}_{s} x=\operatorname{Tr} \tau x
$$

$\operatorname{Tr}_{s}$ vanishes on supercommutators and on odd elements in End $E . \Lambda(N)$ denotes the exterior algebra of $T^{*} N . H=\operatorname{End} E \hat{\otimes} \Lambda(N)$ is the $Z_{2}$ graded tensor product of End $E$ and $\Lambda(N)$. $\operatorname{Tr}_{s}$ can be naturally extended on $H$ as a linear mapping from $H$ into $\Lambda(N)$. Also supercommutators can be defined in $H$, and $\operatorname{Tr}_{s}$ still vanishes on supercommutators.

Let $\nabla$. be a connection on $E$, which preserves the splitting $E=E_{0} \oplus E_{1}$. Its curvature tensor $R$ is an even element of $H$ which we write in the form

$$
R=\left[\begin{array}{cc}
R_{0} & 0 \\
0 & R_{1}
\end{array}\right] .
$$

A representative in cohomology of the Chern character $\operatorname{ch}\left(E_{0}-E_{1}\right)$ is obviously given by

$$
\operatorname{ch}\left(E_{0}-E_{1}\right)=\operatorname{Tr}\left[\exp -\frac{R_{0}}{2 i \pi}\right]-\operatorname{Tr}\left[\exp -\frac{R_{1}}{2 i \pi}\right],
$$

which we also write as

$$
\operatorname{ch}\left(E_{0}-E_{1}\right)=\operatorname{Tr}_{s}\left[\exp -\frac{R}{2 i \pi}\right] .
$$

In [25] Quillen shows how to produce other representatives in cohomology than the obvious (3.5). First, note that $\nabla$ acts naturally on the sections of $\Lambda(N) \otimes E$ by setting for $\eta \in \Lambda(N), A \in E$

$$
\nabla(\eta A)=(d \eta) A+(-1)^{\operatorname{deg} \eta}\left(\eta \wedge d x^{\alpha}\right) \nabla_{e_{\alpha}} A .
$$

Take an odd element $B$ in $H . \nabla+B$ is called a superconnection.

Definition 3.1. The curvature $R^{\prime}$ of $\nabla+B$ is the even element of $H$

$$
R^{\prime}=(\nabla+B)^{2} \text {. }
$$

We now have the result of Quillen [25].

Theorem 3.2 (Quillen [25]). A representative in cohomology of $\operatorname{ch}\left(E_{0}-E_{1}\right)$ is given by

$$
\operatorname{Tr}_{s} \exp \left[-\frac{R^{\prime}}{2 i \pi}\right]
$$


Proof. The proof of Quillen is the same as the proof of the invariance of the Chern character of a bundle when changing the connection. In fact, the theorem holds when $B=0$. We then replace $\tilde{\nabla}+B$ by $\tilde{\nabla}+s B$, and it is easy to show that the cohomology class of (3.9) does not change with $s$, using the fact that $\operatorname{Tr}_{s}$ vanishes on supercommutators.

In particular, if $B$ is an odd element in End $E$, we have Quillen's formula

$$
R^{\prime}=R+\nabla \cdot B+B^{2} .
$$

\section{b) Elementary Properties of a Fibered Manifold}

Let $X$ be an even dimensional compact connected Riemannian manifold of dimension $n=2 l$, which we assume to be oriented and spin. $B^{0}$ denotes a connected compact manifold of dimension $m . M^{0}$ is a compact connected manifold of dimension $n+m, f$ is a submersion of $M^{0}$ onto $B^{0}$. For $y \in B^{0}$, set

$$
G_{y}=f^{-1}(y) \text {. }
$$

$G_{y}$ defines a local trivial fibering of $M^{0} \underset{f}{\rightarrow} B^{0}$. We assume that the fibers $G_{y}$ are modelled on $X$. There is an open covering $\mathscr{U}$ of $B^{0}$ such that for $U \in \mathscr{U}$, $f^{-1}(U) \simeq U \times X$.

There exists a smooth subbundle of $T M$, which we note $T^{H} M^{0}$ such that

$$
T M^{0}=T G \oplus T^{H} M^{0} \text {. }
$$

To determine $T^{H} M^{0}$, it suffices to take the orthogonal bundle of $T G$ in $T M^{0}$ for any Riemannian metric.

It is clear that for any $x \in M, f_{*}$ is a linear isomorphism from $T_{x}^{H} M^{0}$ into $T_{f(x)} B^{0}$. In other words in the sense of $K$-theory

$$
T^{H} M^{0}=f^{*} T B^{0} .
$$

We assume that $B^{0}$ is a Riemannian manifold. Let $g_{B^{0}}$ be the scalar product $T B^{0}$. We lift the scalar product in $T^{H} M^{0}$ by $f^{*}$. Also we assume that $T G$ is endowed with an Euclidean scalar product. By assuming that $T G$ and $T^{H} M^{0}$ are orthogonal in $T M^{0}$, we find that $T M^{0}$ is now endowed with a scalar product. $P_{H}, P_{G}$ denotes the orthogonal projection operators of $T M^{0}$ into $T^{H} M^{0}, T G$.

Let $\nabla^{B^{0}}$ be the Levi-Civita connection of $B^{0}, \nabla^{M^{0}}$ the Levi-Civita connection of $M^{0}$. The connection $\nabla^{B^{0}}$ can be lifted as a connection on $T^{H} M^{0}$ by $f^{*}$. We still note $\nabla^{B^{0}}$ the lifted connection.

Let $\nabla^{G}$ be the connection on $T G$ defined by

$$
X \in T M, \quad Y \in T G, \quad \nabla_{X}^{G} Y=P_{G} \nabla_{X}^{M^{0}} Y .
$$

Let $\nabla$ be the connection on $T M^{0}=T G \oplus T^{H} M^{0}$ defined by

$$
\nabla=\nabla^{G} \oplus \nabla^{B^{0}} \text {. }
$$

Clearly, $\nabla$. preserves the scalar product in $T M^{0}$. Also, for one $y \in B^{0}$, the restriction of $\nabla$ to $T G_{y}$ coincides with the Levi-Civita connection of the Riemannian submanifold $G_{y}$. 
Let $T, R$ be the torsion and curvature tensor of $\nabla$. Set

$$
S=\nabla^{M^{0}}-\nabla \text {. }
$$

For $X \in T M^{0}, S(X)$ is antisymmetric in $\operatorname{End}\left(T M^{0}\right)$.

We first have the technical result.

Theorem 3.3. $T$ takes its values in $T G$. If $X, Y \in T G, T(X, Y)=0$. If $Y, U \in T^{H} M^{0}$, $V, W \in T G$

$$
R(V, W) Y=0, \quad R(Y, V) U=0 .
$$

If $Y \in T M^{0}, V \in T G, S(Y) V \in T^{H} M^{0}$. If $Y, Z \in T^{H} M^{0}, S(Y) Z \in T G$. Finally, $\nabla^{G}, T$ and the $(3,0)$ tensor $\langle S(\cdot) \cdot, \cdot\rangle$ do not depend on $g_{B^{0}}$.

Proof. This simple result is proved in [15, Theorem 1.9].

\section{c) A Connection on an Infinite Dimensional Bundle}

We now assume that the Euclidean bundle $T G$ over $M^{0}$ is oriented and Spin.

Let $F_{+}, F_{-}$be the bundle of spinors associated with $T G$. They are Hermitian $\operatorname{Spin}(n)$ bundles over $M^{0}$. Set $F=F_{+} \oplus F_{-}$. The connection $\nabla$ defines naturally a unitary connection on $F_{ \pm}$. Let $R^{G}$ be the curvature tensor of $\nabla$ of $T G$ which lifts naturally as the curvature tensor of $F_{ \pm}$. Let $\xi$ be a dimensional Hermitian bundle over $M^{0}$, endowed with a unitary connection which we still note $\nabla . L$ denotes the curvature tensor of $\xi$.

Definition 3.4. For $s \in R, y \in B, H_{y}^{s}, H_{ \pm, y}^{s}$ denotes the vectors space of the sections of $F \otimes \xi, F_{ \pm} \otimes \xi$ over $G_{y}$ which are in the s Sobolev space of $G_{y}$.

$H^{s}, H_{ \pm}^{s}$ are infinite dimensional bundles over $B^{0}$. Also $H^{\infty}=H_{+}^{\infty} \oplus H_{-}^{\infty} . H^{\infty}$ is a super vector bundle over $B^{0}$, the even (odd) elements being $H_{+}^{\infty}$ (respectively $H_{-}^{\infty}$ ).

For $X \in T B^{0}$, let $X^{H}$ be the lift in $T^{H} M^{0}$ of $X$.

Definition 3.5. $\tilde{\nabla}$ is the connection on $H_{ \pm}^{\infty}$ such that if $X \in T B^{0}$, if $h$ is a $C^{\infty}$ section of $H_{ \pm}^{\infty}$,

$$
\left(\widetilde{V}_{X} h\right)(x)=\left(\nabla_{X^{H}} h\right)(x) .
$$

Let $\tilde{R}$ be the curvature of $\tilde{\nabla}$.

Proposition 3.6. If $Y, Z \in T B^{0}, \widetilde{R}_{y}(Y, Z)$ is the first order differential operator acting on $H_{ \pm, y}^{\infty}$ by

$$
\widetilde{R}_{y}(Y, Z)=R^{G}\left(Y^{H}, Z^{H}\right) \otimes 1+1 \otimes L\left(Y^{H}, Z^{H}\right)-\nabla_{T\left(Y^{H}, Z^{H}\right)} .
$$

Proof. Clearly,

$$
\tilde{R}_{y}(Y, Z)=\nabla_{X^{H}} \nabla_{Z^{H}}-\nabla_{Z^{H}} \nabla_{Y^{H}}-\nabla_{[Y, Z]^{H}} .
$$

Now $f_{*}\left[Y^{H}, Z^{H}\right]=[Y, Z]$, and so

$$
\widetilde{R}_{y}(Y, Z)=R^{G}\left(Y^{H}, Z^{H}\right) \otimes 1+1 \otimes L\left(Y^{H}, Z^{H}\right)+\nabla_{P_{G}\left[Y^{H}, Z^{H}\right]} .
$$

Now since $\nabla^{B}$ is torsion free, it is easy to see that

$$
T\left(Y^{H}, Z^{H}\right)=-P_{G}\left[Y^{H}, Z^{H}\right] .
$$




\section{d) The Dirac Operator in the Fibers}

We now define the Dirac operator $D . e_{1}, \ldots, e_{n}$ is an orthogonal base of $T G$. $e_{1}, \ldots, e_{n}$ generates the Clifford algebra $c(T G)$ which acts naturally $F \otimes \xi$.

Definition 3.7. D denotes the operator acting on $H_{\infty}$,

$$
D=\sum_{1}^{n} e_{i} \nabla_{e_{i}}
$$

$D_{+}, D_{-}$denote the restriction of $D$ to $H_{+}^{\infty}, H_{-}^{\infty}$. $D$ interchanges $H_{+}^{\infty}, H_{-}^{\infty}$, and so is an odd element in $\operatorname{End}\left(H^{\infty}\right)$. We will write

$$
D=\left[\begin{array}{cc}
0 & D_{-} \\
D_{+} & 0
\end{array}\right]
$$

$D_{y}$ acts fiberwise on $H_{y}^{\infty}$. We want to calculate the Chern character

$$
\operatorname{ch}\left(\operatorname{Ker} D_{+, y}-\operatorname{Ker} D_{-, y}\right) \text {. }
$$

\section{e) Index Theory and Infinite Dimensional Chern Weil Theory}

The Fredholm operator $D$ on $H^{\infty}$ introduces naturally a rigidity in the description of $H^{\infty} . D$ should be viewed as creating a "connection" (in the naive sense!) between $H_{+}^{\infty}$ and $H_{-}^{\infty} . \tilde{\nabla}+D$ is a superconnection in the sense of Quillen [25].

Let $I$ be the curvature of $\tilde{\nabla}+D$, i.e. set

$$
I=(\tilde{\nabla}+D)^{2} \text {. }
$$

Let $\Delta^{H}$ be the Bochner Laplacian acting fiberwise on $H_{ \pm}^{\infty}$.

We first compute $I . f_{1}, \ldots, f_{n}$ denotes a base of $T_{y} B, d y^{1} \ldots d y^{m}$ is the corresponding dual base. We identify $f_{\alpha}, d y^{\alpha}$ to the corresponding elements in $T^{H} M^{0}, T^{H^{*}} M^{0}$.

Theorem 3.8. $I$ is the even element in $\Lambda\left(B^{0}\right) \hat{\otimes} \operatorname{End} H^{\infty}$,

$$
\begin{aligned}
I= & D^{2}-\frac{1}{2} d y^{\alpha} d y^{\beta} \nabla_{T\left(f_{\alpha}, f_{\beta}\right)}-d y^{\alpha} e_{i} \nabla_{T\left(f_{\alpha}, e_{t}\right)} \\
& +\frac{1}{2} d y^{\alpha} d y^{\beta}\left[R^{G}\left(f_{\alpha}, f_{\beta}\right) \otimes 1+1 \otimes L\left(f_{\alpha}, f_{\beta}\right)\right] \\
& +d y^{\alpha} e_{i}\left[R^{G}\left(f_{\alpha}, e_{i}\right) \otimes 1+1 \otimes L\left(f_{\alpha}, e_{i}\right)\right] .
\end{aligned}
$$

Proof. The computation of $I$ is easy and given in [15, Theorem 2.5].

$I$ acts on the $Z_{2}$ graded bundle $H^{\infty} \otimes \Lambda\left(B^{0}\right)$ by assuming that if $h \in H^{\infty}$, $\eta \in \Lambda\left(B^{0}\right), I(h \eta)=(I h) \eta$. The principal symbol of $I_{y}$ is $|\xi|^{2}$. It follows using standard elliptic theory that for $s>0, e^{-\frac{s I_{y}}{2}}$ is given by a smooth kernel $P_{s}^{y}\left(x, x^{\prime}\right)$ (with $\left.x, x^{\prime} \in G_{y}\right), P_{s}^{y}\left(x, x^{\prime}\right)$ is a linear mapping from $(F \otimes \xi)_{x^{\prime}}$ into $(F \otimes \xi)_{x} \otimes \Lambda_{y}\left(B^{0}\right)$ which is even.

In particular, for $x \in G_{y}, P_{s}^{y}(x, x)$ is an even element of $\operatorname{End}(F \otimes \xi)_{x} \hat{\otimes} \Lambda_{y}\left(B^{0}\right)$, so that $\operatorname{Tr}_{s} P_{s}^{y}(x, x)$ is even in $\Lambda_{y}\left(B^{0}\right)$.

In the sequel, if $E$ is a fiber bundle, with a connection and curvature $C, \overline{c h} E$ denotes the cohomology class of $\operatorname{Tr}\left[\exp -\frac{C}{2}\right] \cdot \overline{c h} E$ is the normalized Chern
character of $E$. 
$d x$ denotes the volume element in $G_{y}$.

We now have the key result of [15].

Theorem 3.9. The $C^{\infty}$ form over $B^{0}$

$$
\int_{G_{y}} \operatorname{Tr}_{s} P_{1}^{y}(x, x) d x
$$

is closed and is a representative in cohomology of $\overline{\operatorname{ch}}\left(\operatorname{Ker} D_{+}-\operatorname{Ker} D_{-}\right)$.

Proof. The proof of the theorem consists in showing that (3.13) is closed, invariant (in cohomology) under deformations of $\tilde{\nabla}$. Connections associated with pseudodifferential operators have to be considered. The reader is referred to [15] for more details.

\section{f) The Levi-Civita Superconnection}

The idea of our first proof in [15] is to scale the metric of the fibers adequately. However, in the same way as for the ordinary Index Theorem, the Levi-Civita connection is the only one which works, in our case, it will be the Levi-Civita superconnection. In (3.58), we will give an interpretation of this very similar to (2.33).

Namely, let $E$ be the odd element of $c(T G) \hat{\otimes} \Lambda\left(B^{0}\right)$,

$$
\begin{aligned}
E= & e_{i}\left[\frac{1}{2}\left\langle S\left(e_{i}\right) e_{j}, f_{\alpha}\right\rangle e_{j} d y^{\alpha}+\frac{1}{4}\left\langle S\left(e_{i}\right) f_{\alpha}, f_{\beta}\right\rangle d y^{\alpha} d y^{\beta}\right] \\
& +\frac{1}{2}\left\langle S\left(f_{\alpha}\right) e_{i}, f_{\beta}\right\rangle d y^{\alpha} e_{i} d y^{\beta} .
\end{aligned}
$$

By Theorem 3.3, $\left\langle S\left(e_{i}\right) e_{j}, e_{k}\right\rangle=\left\langle S\left(f_{\alpha}\right) e_{j}, e_{k}\right\rangle=\left\langle S\left(f_{\alpha}\right) f_{\beta}, f_{\gamma}\right\rangle=0$.

The Levi-Civita superconnection $\tilde{V}^{L}$ on $H^{\infty}$ is defined by $\tilde{V}^{L}=\tilde{V}+E$. Of course, the superconnection $\widetilde{\nabla}^{L}+D$ is given by

$$
\begin{aligned}
\tilde{V}^{L}+D= & e_{i}\left[\nabla_{e_{i}}+\frac{1}{2}\left\langle S\left(e_{i}\right) e_{j}, f_{\alpha}\right\rangle e_{j} d y^{\alpha}+\frac{1}{4}\left\langle S\left(e_{i}\right) f_{\alpha}, f_{\beta}\right\rangle d y^{\alpha} d y^{\beta}\right] \\
& +d y^{\alpha}\left[\nabla_{f_{\alpha}}+\frac{1}{2}\left\langle S\left(f_{\alpha}\right) e_{i}, f_{\beta}\right\rangle e_{i} d y^{\beta}\right] .
\end{aligned}
$$

Note that $\tilde{\nabla}^{L}+D$ appears formally as the usual Dirac operator of $M^{0}$ calculated with the Levi-Civita connection of $T M^{0}$ in which the horizontal Clifford variables $f_{\alpha}$ are replaced by the Grassmann variables $d y^{\alpha}$. This justifies the terminology which we choose. $\tilde{V}^{L}+D$ should be viewed as a generalized Dirac operator acting on the whole space $M$. Note that we use here the full strength of Quillen's formalism, since $E$ is a very general odd element of End $H^{\infty} \hat{\otimes} \Lambda\left(B^{0}\right)$.

Let $I^{L}$ be the curvature of $\tilde{V}^{L}+D$, i.e. $I^{L}=\left(\tilde{V}^{L}+D\right)^{2}$. Let $P_{s}^{L, y}\left(x, x^{\prime}\right)$ be the heat equation semi-group associated with $e^{-\frac{s I^{L}}{2}}$. Since $\tilde{\nabla}^{L}+D$ is a mild perturbation of $\tilde{\nabla}+D$, we still show in $[15]$ that

$$
\int \operatorname{Tr}_{s}\left[P_{1}^{L, y}(x, x)\right] d x
$$

is a representative of $\overline{\operatorname{ch}}\left(\operatorname{Ker} D_{+, y}-\operatorname{Ker} D_{-, y}\right)$.

Let $K(x)$ be the scalar curvature of the fiber $G_{f(x)}$ at $x$.

The key to the explicit computation of $\overline{\operatorname{ch}}\left(\operatorname{Ker} D_{+}-\operatorname{Ker} D_{-}\right)$is the following extension of Lichnerowicz's formula (2.12), which includes (2.37) as a special case. 
Theorem 3.10. $I^{L}$ is given by

$$
\begin{aligned}
I^{L}= & -\left(\nabla_{e_{i}}+\frac{1}{2}\left\langle S\left(e_{i}\right) e_{j}, f_{\alpha}\right\rangle e_{j} d y^{\alpha}+\frac{1}{4}\left\langle S\left(e_{i}\right) f_{\alpha}, f_{\beta}\right\rangle d y^{\alpha} d y^{\beta}\right)^{2} \\
& +\frac{K}{4}+\frac{1}{2} e_{i} e_{j} \otimes L\left(e_{i}, e_{j}\right)+\frac{1}{2} d y^{\alpha} d y^{\beta} \otimes L\left(f_{\alpha}, f_{\beta}\right)+e_{i} d y^{\alpha} \otimes L\left(e_{i}, f_{\alpha}\right) .
\end{aligned}
$$

Proof. The proof in [15, Theorem 3.5] is as follows. We consider the usual Dirac operator $D^{L}$ on $M$ associated with the Levi-Civita connection of $M$. We can then write Lichnerowicz's formula (2.12) for $D^{L}$. We now scale the metric on $B^{0}$ by the factor $1 / \varepsilon$. This is equivalent to replacing the horizontal Clifford variables $f_{\alpha}$ by $\sqrt{\varepsilon} f_{\alpha}$. Let $D^{L, \varepsilon}$ be the new Dirac operator on $M$ associated with this metric. We again write Lichnerowicz's formula for $D^{L, \varepsilon}$. We now observe the following identities

$$
\begin{aligned}
& \sqrt{\varepsilon} f_{\alpha} \sqrt{\varepsilon} f_{\beta}=(\sqrt{\varepsilon})^{2} f_{\alpha} f_{\beta}, \quad \alpha \neq \beta, \\
& \sqrt{\varepsilon} f_{\alpha} \sqrt{\varepsilon} f_{\alpha}=-\varepsilon .
\end{aligned}
$$

In the first line of (3.28), $\sqrt{\varepsilon}$ appears with the power 2 corresponding to the length of $f_{\alpha} f_{\beta}$ in the Clifford algebra. On the contrary in the second line, $\sqrt{\varepsilon}$ appears with power 2 , which is strictly larger than the length of -1 (which is 0 ). In other words, to calculate $I^{L}$, i.e. to obtain what is the result of the calculation where the Clifford variables $f_{\alpha}$ are replaced by the Grassmann variables $d y^{\alpha}$, it suffices to identify in Lichnerowicz's formula those terms in the Clifford algebra of $T B^{0}$ where $\sqrt{\varepsilon}$ appears with a power exactly equal to the length of these terms. So we obtain (3.27).

Remark 1. The idea of blowing up the metric on $B^{0}$ plays a key role in both proofs in [15] of the Index Theorem for families.

\section{g) The Index Theorem for Families: A First Proof}

To simplify the exposition, we assume in this paragraph that $\xi$ is the trivial bundle, so that $L=0$. We first scale the metric in the fiber by a factor $\frac{1}{t} . D$ is then changed into $\sqrt{t} D$. An easy computation in [15] shows that $I^{L}$ is changed into $I^{L, t}$ given by

$$
I^{L, t}=-t\left(\nabla_{e_{i}}+\frac{1}{2 t}\left\langle S\left(e_{i}\right) e_{j}, f_{\alpha}\right\rangle \sqrt{t} e_{j} d y^{\alpha}+\frac{1}{4 t}\left\langle S\left(e_{i}\right) f_{\alpha}, f_{\beta}\right\rangle d y^{\alpha} d y^{\beta}\right)^{2}+\frac{t K}{4} .
$$

Let $P_{s}^{L, t, y}\left(x, x^{\prime}\right)$ be the kernel of $e^{-\frac{s I^{L, t}}{2}}$. Still we have

$$
\overline{\operatorname{ch}}\left(\operatorname{Ker} D_{+, y}-\operatorname{Ker} D_{-, y}\right)=\int_{G_{y}} \operatorname{Tr}_{s}\left[P_{1}^{L, t, y}(x, x)\right] d x .
$$

To study the asymptotics of (3.30) as $t \downarrow \downarrow 0$, we need an analogue of (2.21).

We use the notation of Sect. 2 in a given fiber $G_{y}$. Parallel transport will be calculated with respect to $\nabla$ (which coincides with the Levi-Civita connection of $G_{y}$ on $T G)$ and is the identity on $T^{H} M^{0}$ identified with $T_{y} B^{0}$. 
$E_{x_{0}, x_{0}}^{t}$ still denotes the expectation operator for the scaled Brownian bridge $x_{s}$ in the fiber $G_{y}$ with $x(0)=x(1)=x_{0} . p_{t}\left(x, x^{\prime}\right)$ is the heat kernel in $G_{y}$ for the LaplaceBeltrami operator of $G_{y}$. We will write $x_{s}^{t}, \tau_{0}^{s, t}$ instead of $x_{s}, \tau_{0}^{s}$.

Definition 3.11. On $\left.\left(\mathscr{C}([0,1]) ; G_{y}\right) ; E_{x_{0}, x_{0}}^{t}\right), U_{s}^{t}$ denotes the solution of the Stratonovitch differential equation,

$$
\begin{aligned}
d U_{s}^{t} & =U_{s}^{t}\left[\frac{1}{2 t}\left\langle\left(\tau_{0}^{s, t} S\left(d x_{s}^{t}\right)\right) e_{i}, f_{\alpha}\right\rangle \sqrt{t} e_{i} d y^{\alpha}+\frac{1}{4 t}\left\langle S\left(d x_{s}^{t}\right) f_{\alpha}, f_{\beta}\right\rangle d y^{\alpha} d y^{\beta}\right], \\
U_{0}^{t} & =I .
\end{aligned}
$$

$U_{s}^{t}$ takes its values in End $F_{x_{0}} \hat{\otimes} \Lambda_{y}\left(B^{0}\right)$ and is obviously even.

As shown in [15, Theorem 4.2], the analogue of (2.21) is as follows.

Proposition 3.12. The following identity holds

$$
\begin{aligned}
\operatorname{Tr}_{s} & {\left[P_{1}^{L, y, t}\left(x_{0}, x_{0}\right)\right] } \\
& =p_{t}\left(x_{0}, x_{0}\right) E_{x_{0}, x_{0}}^{t}\left[\exp \left\{-t \int_{0}^{1} \frac{K\left(x_{s}^{t}\right)}{8} d s\right\} \operatorname{Tr}_{s}\left[U_{1}^{t} \tau_{0}^{1, t}\right]\right] .
\end{aligned}
$$

It is very interesting to interpret (3.27), (3.31), and (3.32). $\operatorname{In} \operatorname{Tr}_{s}\left[U_{1}^{t} \tau_{0}^{1, t}\right]$, the scalar term is the one which calculates the $Z$ valued index of $D_{+}$. In (3.31) the expression before $e_{i} d y^{\alpha}$ measures how much the fiber $G$ is not totally geodesic in $M$. The expression before $d y^{\alpha} d y^{\beta}$ measures the lack of integrability of the bundle $T^{H} M$ in $M$.

The reader will have noted the singularity $\frac{1}{t}$ in (3.32). This singularity is not killed by $d x_{s}^{t}$ which is of the order $\sqrt{t}$.

The key to the proof of the Index Theorem is the possibility of integrating (3.31) explicitly. Let $\mathscr{A}$ be the set of $(n, n)$ antisymmetric matrices on $T G$ identified with the vector subspace of $c(T G)$ spanned by $\left(e_{i} e_{j}\right)_{i<j}$. Let $\mathscr{F}$ be the subspace of $c(T G) \hat{\otimes} \Lambda\left(B^{0}\right)$ spanned by $e_{i} d y^{\alpha}, d y^{\alpha} d y^{\beta}$. We now do the key observation that $\mathscr{F}$ is a Heisenberg algebra, such that $\left\{d y^{\alpha} d y^{\beta}\right\}$ spans the center of $\mathscr{F}$, with the commutation relations

$$
\left[e_{i} d y^{\alpha}, e_{j} d y^{\beta}\right]=2 \delta_{i j} d y^{\alpha} d y^{\beta} .
$$

Theorem 3.13. $U_{s}^{t}$ is exactly given by

$$
\begin{aligned}
U_{s}^{t}= & \exp \left\{\frac{1}{4 t} \int_{0}^{s}\left\langle S\left(d x_{h}^{t}\right) f_{\alpha}, f_{\beta}\right\rangle d y^{\alpha} d y^{\beta}+\frac{1}{2 t} \int_{0}^{s}\left\langle\left(\tau_{0}^{h, t} S\left(d x_{h}^{t}\right)\right) e_{i}, f_{\alpha}\right\rangle \sqrt{t} e_{i} d y^{\alpha}\right. \\
& \left.+\frac{1}{4} \int_{0 \leqq h \leqq h^{\prime} \leqq s}\left\langle\frac{P_{G} \tau_{0}^{h, t} S\left(d x_{h}^{t}\right) f_{\alpha}}{\sqrt{t}}, \frac{P_{G^{\prime}} \tau_{0}^{h^{\prime}, t} S\left(d x_{h^{\prime}}^{t}\right) f_{\beta}}{\sqrt{t}}\right\rangle d y^{\alpha} d y^{\beta}\right\} .
\end{aligned}
$$

Proof. $U_{s}^{t}$ is the solution of an equation of the form

$$
d U_{s}^{t}=U_{s}^{t} d F_{s}, \quad U_{0}^{t}=I,
$$

where $F_{s}$ a process valued in the Heisenberg algebra $\mathscr{F}$. An elementary application of the Campbell-Hausdorf's formula shows that

$$
U_{s}^{t}=\exp \left\{F_{s}+\frac{1}{2} \int_{0}^{s}\left[F_{h}, d F_{h}\right]\right\} .
$$

We then use the commutation relations (3.33). 
We now use the same notations as in Sect. 2. The analogue of (2.23) is here

$$
\operatorname{Tr}_{s} P_{1}^{L, y, t}\left(x_{0}, x_{0}\right)=\int \frac{\operatorname{Tr}_{s}\left[U_{1}^{t} \tau_{0}^{1, t}\right] H\left(\sqrt{t} d w^{1}\right) d P_{1}\left(w^{1}\right)}{(\sqrt{2 \pi t})^{n}}+o\left(t^{k}\right) .
$$

This is not as obvious as (2.23), because of the singularity $\frac{1}{t}$ in (3.34). However, as shown in [15, Proposition 4.3], since (3.34) is in fact a finite power series, large deviations as used in [10] shows that the singularity in (3.34) is not strong enough to prevent localization.

Due to (2.25), we have

$$
\tau_{0}^{1, t}=\exp \left\{-\frac{1}{8} \int_{0}^{1}\left\langle R_{x_{0}}\left(d w^{\prime 1}, w^{\prime 1}\right) e_{i}, e_{j}\right\rangle \sqrt{t} e_{i} \sqrt{t} e_{j}+o(t)\right\},
$$

where $o(t)$ is calculated in the Lie algebra $\mathscr{A}$. Also a form of Stokes' formula shows that as $t \downarrow \downarrow 0$,

$$
\begin{aligned}
\frac{1}{t} \int_{0}^{1}\left\langle S\left(d x_{s}^{t}\right) f_{\alpha}, f_{\beta}\right\rangle & \rightarrow \int_{0}^{1}\left\langle\nabla_{w_{s}^{1}} S_{x_{0}}\left(d w_{s}^{1}\right) f_{\alpha}, f_{\beta}\right\rangle, \\
\int_{0}^{1} \frac{\left\langle\tau_{0}^{s, t} S\left(d x_{s}^{t}\right) e_{i}, f_{\alpha}\right\rangle}{t} & \rightarrow \int_{0}^{1}\left\langle\nabla_{w^{1}} S_{x_{0}}\left(d w^{1}\right) e_{i}, f_{\alpha}\right\rangle .
\end{aligned}
$$

Using the fact that if $\alpha \in c(T G)$ has length $<n, \operatorname{Tr}_{s} \alpha=0$ and also

$$
\operatorname{Tr}_{s} e_{1} \ldots e_{n}=(-2 i)^{l} \text {, }
$$

we finally obtain in [15, Theorem 4.12].

Theorem 3.14. As $t \downarrow \downarrow 0$, the differential form $\operatorname{Tr}_{s}\left[P_{1}^{L, y, t}\left(x_{0}, x_{0}\right)\right] d x$ converges to a differential form which is the term of degree $n$ in the Grassmann variables $d x^{1}, \ldots, d x^{n}$ in $\mathscr{L}\left(x_{0}\right)$ given by

$$
\begin{aligned}
\mathscr{L}\left(x_{0}\right)= & (-i)^{l} \int_{W} \exp \left\{-\frac{1}{8 \pi} \int_{0}^{1}\left\langle R\left(d w^{1}, w^{1}\right) e_{i}, e_{j}\right\rangle d x^{i} d x^{j}\right. \\
& +\frac{1}{4} \int_{0}^{1}\left\langle\nabla_{w^{1}} S\left(d w^{1}\right) f_{\alpha}, f_{\beta}\right\rangle d y^{\alpha} d y^{\beta} \\
& +\frac{1}{\sqrt{\pi}} \int_{0}^{1}\left\langle\frac{\nabla_{w_{s}^{1}}}{2} S\left(d w_{s}^{1}\right) e_{i}, f_{\alpha}\right\rangle d x^{i} d y^{\alpha} \\
& \left.+\frac{1}{4} \int_{0}^{1}\left\langle P_{G} S\left(w_{s}^{1}\right) f_{\alpha}, P_{G} S\left(d w_{s}^{1}\right) f_{\beta}\right\rangle d y^{\alpha} d y^{\beta}\right\} .
\end{aligned}
$$

In the sequel we identify a $(n+m, n+m)$ antisymmetric element $B$ of End $T M^{0}$ with the 2-form $X, Y \rightarrow\langle X, B Y\rangle$.

Recall that $\int_{G} \mathscr{L}\left(x_{0}\right)$ is a representative of $\overline{\operatorname{ch}}\left(\operatorname{Ker} D_{+}-\operatorname{Ker} D_{-}\right)$. By changing the normalization so as to obtain $\operatorname{ch}\left(\operatorname{Ker} D_{+}-\operatorname{Ker} D_{-}\right)$, we get

$$
\begin{aligned}
\mathscr{L}^{\prime}\left(x_{0}\right)= & \int_{W} \exp \left\{\frac { i } { 4 \pi } \left(\int _ { 0 } ^ { 1 } \left\langleR\left(w_{s}^{1}, d w_{s}^{1}\right)+2 \nabla_{w_{s}^{1}} S\left(d w_{s}^{1}\right)\right.\right.\right. \\
& \left.\left.-\int_{0}^{1}\left\langle P_{G} S\left(w_{s}^{1}\right) f_{\alpha}, P_{G} S\left(d w_{s}^{1}\right) f_{\beta}\right\rangle d y^{\alpha} d y^{\beta}\right)\right\} d P_{1}\left(w^{1}\right) .
\end{aligned}
$$


Still (3.41) is not very nice. However, we have

Theorem 3.15. For $Y, Z \in T_{x_{0}} M^{0}$,

$$
\begin{aligned}
\int_{0}^{1}\left\langle Y,\left(R\left(w_{s}^{1}, d w_{s}^{1}\right)+2 \nabla_{w_{s}^{1}} S\left(d w_{s}^{1}\right)\right) Z\right\rangle-2 \int_{0}^{1}\left\langle P_{G} S\left(w_{s}^{1}\right) Y, P_{G} S\left(d w_{s}^{1}\right) Z\right\rangle \\
=-\int_{0}^{1}\left\langle R(Y, Z) w_{s}^{1}, d w_{s}^{1}\right\rangle .
\end{aligned}
$$

Proof. The proof in [15, Theorem 4.14] uses the Bianchi identities and integration by parts repeatedly. In Theorem 3.18, we reproduce the proof of (3.42), but we interpret (3.42) as being a form of the identity (1.34) (as used in Proof No. 2 of Theorem 1.9) i.e. as a symmetry identity for the Levi-Civita curvature of an infinite dimensional manifold.

We have then found that

$$
\mathscr{L}^{\prime}\left(x_{0}\right)=\int_{W} \exp \left\{\frac{-i}{4 \pi} \int_{0}^{1}\left\langle R^{G}(\cdot, \cdot) w_{s}^{1}, d w_{s}^{1}\right\rangle\right\} d P_{1}\left(w^{1}\right) .
$$

By a well-known formula of P. Lévy [12], we know that if $\hat{A}$ is the Hirzebruch polynomial,

$$
\mathscr{L}^{\prime}\left(x_{0}\right)=\hat{A}\left(\frac{R^{G}}{2 \pi}\right) .
$$

Using (3.30), (3.40), (3.44), we have then given a first proof of the Index Theorem for families.

Theorem 3.16. The following identity holds

$$
\operatorname{ch}\left(\operatorname{Ker} D_{+}-\operatorname{Coker} D_{+}\right)=\int_{G} \hat{A}\left(\frac{R^{G}}{2 \pi}\right) \operatorname{ch} \xi .
$$

\section{h) A Second Proof}

We now briefly describe the second proof of the Index Theorem in [15]. Namely, assume that $B^{0}$ is even dimensional and Spin (this is no restriction).

Let $F_{+}^{\prime}, F_{-}^{\prime}$ be the bundles of spinors over $B$. By lifting $F_{+}^{\prime}, F_{-}^{\prime}$ to $M$, we find that $M$ is Spin, and that if $C_{+}, C_{-}$are the bundles of spinors over $M$, then

$$
C_{+}=\left(F_{+} \otimes F_{+}^{\prime}\right) \oplus\left(F_{-} \otimes F_{-}^{\prime}\right), \quad C_{--}=\left(F_{+} \otimes F_{-}^{\prime}\right) \oplus\left(F_{-} \otimes F_{+}^{\prime}\right) .
$$

Let $D^{\prime t}$ be the Dirac operator acting on the sections of $C_{ \pm}$,

$$
D^{\prime t}=e_{i} \nabla_{e_{i}}+\sqrt{t} f_{\alpha} \nabla_{f_{\alpha}} .
$$

We write $D^{\prime}$ instead of $D^{\prime 1}$. Let $R^{B^{0}}$ be the curvature tensor of $B^{0}$. We now directly prove in [15, Theorem 5.3].

Theorem 3.17. The index of $D_{+}^{\prime}$ is given by

$$
\text { Ind } D_{+}^{\prime}=\left(-\frac{i}{\pi}\right)^{m / 2} \int_{B^{0}} \hat{A}\left(\frac{i R^{B^{0}}}{2 \pi}\right) \wedge \int_{G} \operatorname{Tr}_{s}\left[P_{1}^{y}(x, x)\right] d x \text {. }
$$


Proof. The idea of the proof is to start with the equality

$$
\text { Ind } D_{+}^{\prime}=\operatorname{Tr}_{s}\left[e^{-\frac{\left(D^{\prime}\right)^{2}}{2}}\right] \text {. }
$$

This is not as obvious as it may seem since $D^{\prime t}$ is not self-adjoint. However, by deforming continuously $D^{\prime t}$ into the Levi-Civita Dirac operator, it is not difficult to prove that the right-hand side of (3.48) does not change, so that (3.48) holds (see Remark 2 in Sect. 2).

By making $t \downarrow \downarrow 0$ in (3.48), it is then possible to prove - using precise estimates on the heat kernels obtained via the partial Malliavin calculus (see Bismut and Michel [16]) - that (3.47) holds.

Also we know that classically

$$
\text { Ind } D_{+}^{\prime}=\left(-\frac{i}{\pi}\right)^{(n+m) / 2} \int_{M^{0}} \hat{A}\left(\frac{i R}{2}\right) \text {. }
$$

More generally, if $\xi^{\prime}$ is any Hermitian bundle over $B^{0}$ which is lifted to $M^{0}$, we can prove that if $D^{\prime \xi}$ is the Dirac operator on $C \otimes \xi$, then if $R^{B^{0}}$ is the Levi-Civita curvature tensor of $B^{0}$,

$$
\operatorname{Ind} D_{+}^{\xi \xi}=\left(-\frac{i}{\pi}\right)^{m / 2} \int_{B^{0}} \hat{A}\left(\frac{i R^{B^{0}}}{2}\right) \int_{G} \operatorname{Tr}_{s}\left[P_{1}^{y}(x, x) d x\right] \overline{\operatorname{ch}} \xi^{\prime},
$$

and also

$$
\operatorname{Ind} D_{+}^{\prime \xi}=\left(-\frac{i}{\pi}\right)^{(n+m) / 2} \int_{B^{0}} \hat{A}\left(\frac{i R}{2}\right) \overline{\operatorname{ch}} \xi^{\prime} .
$$

Now recall that

$$
K\left(B^{0}\right) \otimes_{Z} R=H^{* \text { even }}\left(B^{0}, R\right) .
$$

Using Poincaré duality and (3.50), (3.51), we find that in cohomology

$$
\hat{A}\left(\frac{i R^{B^{0}}}{2}\right) \int_{G} \operatorname{Tr}_{s}\left[P_{1}^{y}(x, x)\right] d x=\left(-\frac{i}{\pi}\right)^{n / 2} \hat{A}\left(\frac{i R}{2}\right) .
$$

Since $T M^{0}=T B^{0} \oplus T G$, we have

$$
\hat{A}\left(\frac{i R}{2}\right)=\hat{A}\left(\frac{i R^{B^{0}}}{2}\right) \hat{A}\left(\frac{i R^{G}}{2}\right) .
$$

Since $\hat{A}\left(\frac{i R^{B^{0}}}{2 \pi}\right)$ is stable, we get from (3.53), (3.54) that in cohomology

$$
\int_{G_{y}} \operatorname{Tr}_{s}\left[P_{1}(x, x)\right] d x=\left(-\frac{i}{\pi}\right)^{n / 2} \int_{G_{y}} \hat{A}\left(\frac{i R^{G}}{2}\right) .
$$

The theorem is proved again.

Note that instead of $D^{\prime t}$, we might have as well used the Dirac operator calculated with the Levi-Civita connection. 
i) Superconnections, Quillen's Formalism, and Integration Along the Fiber in the Loop Space

We now hope to convince the reader that the preceding proofs are the infinite dimensional analogues of the two proofs of Theorem 1.9. $M, X$ are defined as in Sect. 2. $B, Y$ are the analogues of $M, X$ for the manifold $B^{0}$. Clearly,

$$
f_{*} X=Y \text {. }
$$

Also

$$
M^{0}=M^{X}, \quad B^{0}=B^{Y} .
$$

The assumptions of Sect. 1d) are then formally verified. We will now use the notations of Sect. 1d). $M^{Y}=f^{-1}\left(B^{Y}\right)$ is here the set of smooth loops in $M^{0}$ whose projection is constant in $B^{0}$. For $y \in B, C_{y}$ is the set of loops in $M$ which project on $y$, and the two definitions of $G$ in (1.49) and (3.10) coincide.

On the algebra of even forms on $B$, let $*$ be the homomorphism defined by

$$
*\left(d y^{\alpha} d y^{\beta}\right)=-2 d y^{\alpha} d y^{\beta} .
$$

We then claim that formally

$$
\begin{aligned}
& *\left[\int_{G}\left[\operatorname{Tr}_{s} P_{1}^{L, y, t}(x, x)\right] d x\right]=\frac{\left(\prod_{1}^{+\infty} k^{2}\right)^{l} i^{l} j^{*}}{(2 \pi)^{l}} \int_{C} \exp \left\{-\frac{\left(d+i_{X}\right) X}{2 t}\right\}, \\
& *\left[\int_{G}\left[\operatorname{Tr}_{s} P_{1}^{y}(x, x)\right] d x\right]=\frac{\left(\prod_{1}^{+\infty} k^{2}\right)^{l} i^{l} j^{*}}{(2 \pi)^{l}} \int_{C} \exp \left\{-\frac{\left(d+i_{X}\right) X}{2}-\left(d+i_{X}\right) \frac{\langle T \wedge \theta\rangle}{2}\right\} .
\end{aligned}
$$

The best justification we have for (3.58) is as follows.

By formulas (2.18) and (2.33), we know that formally

$$
\text { Ind } \begin{aligned}
D_{+}^{\prime} & =C \int_{M} \exp -\frac{\left(d+i_{X}\right) X}{2} \\
& =C \int_{M} \exp \left\{-\frac{\left(d+i_{X}\right) X}{2}-\frac{\left(d+i_{X}\right)(\langle T \wedge \theta\rangle}{2}\right\} .
\end{aligned}
$$

We now scale the metric of $B^{0}$ by a factor $\frac{1}{t}$.

This is exactly what we did in the Proof of Theorem 3.17. Now combining Proof No. 2 of Theorem 1.3 and Proof No. 2 of Theorem 1.9, this is also what is done in Sect. 1. It is then possible to deduce formally (3.58) from (3.59) by introducing an auxiliary bundle $\xi^{\prime}$ on $B^{0}$ and by proceeding as in Proof No. 2 of Theorem 1.9.

In particular, formulas (3.31), (3.34) have an easy interpretation. $U_{1} \tau_{0}^{1}$ is indeed an infinitesimal version of parallel transportation along $x$, for the Levi-Civita connection of $M^{0}$. Using the remark of Atiyah contained in formula (2.20), we see that $\operatorname{Tr}_{s}\left[U_{1} \tau_{0}^{1}\right]$ exactly constructs the right factor to be integrated along the fibers of $M^{Y}$. 
Equation (3.58) is not only a nice remark, since it permitted us to define the Levi-Civita superconnection, and predict the computations with great precision. As an example of this, we will now relate Theorem 3.15 with the appearance of the curvature tensor $R^{L}$ in Proof No. 2 of Theorem 1.9.

Recall that $\nabla^{M^{0}}$ is the Levi-Civita connection on $M^{0}$. Let $R^{M^{0}}$ denote the corresponding curvature tensor. Similarly, $\nabla^{M}$ (respectively $\nabla^{L}$ ) is the Levi-Civita connection on $M$ (respectively $M^{Y}$ ), $R^{M}$ (respectively $R^{L}$ ) its curvature tensor. The connection $\nabla$ and its curvature $R$ can be extended to $M$ in the obvious way. Here again, we identify $T_{x}^{H} M^{0}$ with $T_{f(x)} B^{0}$. If $X^{\prime} \in T M^{Y}, P_{H} X^{\prime}$ is a constant vector.

We now have

Theorem 3.18. For $T, U \in T M^{Y}$

$$
\nabla_{T}^{M} U=\nabla_{T}^{L} U+P_{H}[S(T) U]-\int_{0}^{1} P_{H} S\left(T_{s}\right) U_{s} d s,
$$

or equivalently

$$
\nabla_{T} U=\nabla_{T}^{L} U-S(T) U+P_{H} S(T) U-\int_{0}^{1} P_{H} S\left(T_{s}\right) U_{s} d s .
$$

Also if $T, U, V, W \in T_{x} M^{Y}$,

$$
\begin{aligned}
\int_{0}^{1}\left\langle R^{M^{0}}(T, U) V, W\right\rangle d s= & \left\langle R^{L}(T, U) V, W\right\rangle+\int_{0}^{1}\left[\left\langle P_{H} S\left(T_{s}\right) V_{s}, P_{H} S\left(U_{s}\right) W_{s}\right\rangle\right. \\
& \left.-\left\langle P_{H} S\left(U_{s}\right) V_{s}, P_{H} S\left(T_{s}\right) W_{s}\right\rangle\right] d s \\
& -\left(\left\langle\int_{0}^{1} P_{H} S\left(T_{s}\right) V_{s} d s, \int_{0}^{1} P_{H} S\left(U_{s}\right) W_{s} d s\right\rangle\right. \\
& \left.-\left\langle\int_{0}^{1} P_{H} S\left(U_{s}\right) V_{s} d s, \int_{0}^{1} P_{H} S\left(T_{s}\right) W_{s} d s\right\rangle\right) .
\end{aligned}
$$

In particular, if $x_{0} \in M^{0}$, if $x_{s}$ is the constant path $x_{s}=x_{0}$, if $s \rightarrow w_{s}^{\prime 1}$ is a $C^{\infty}$ mapping from $[0,1]$ into $T_{x_{0}} G_{y}$ such that $w_{0}^{\prime 1}=w_{1}^{\prime 1}, \int_{0}^{1} w_{h}^{\prime 1} d h=0$, if $Y^{\prime}, Z^{\prime} \in T_{x_{0}} M^{0}$, then

$$
\begin{aligned}
\left\langle R_{x_{0}}^{L}\left(Y^{\prime}, Z^{\prime}\right) w^{\prime 1}, \frac{d w^{\prime 1}}{d s}\right\rangle= & \int_{0}^{1}\left\langle R\left(Y^{\prime}, Z^{\prime}\right) w_{s}^{\prime 1}, d w_{s}^{\prime 1}\right\rangle \\
= & \int_{0}^{1}\left\langle\left(R\left(w_{s}^{\prime 1}, d w_{s}^{\prime 1}\right)+2 V_{w_{s}^{\prime}} S\left(d w_{s}^{\prime 1}\right)\right) Y^{\prime}, Z^{\prime}\right\rangle \\
& -2 \int_{0}^{1}\left\langle P_{G}\left[S_{x_{0}}\left(d w_{s}^{\prime 1}\right) Y^{\prime}\right], P_{G}\left[S_{x_{0}}\left(w_{s}^{\prime 1}\right) Z^{\prime}\right]\right\rangle \\
= & \left\langle R_{x_{0}}^{L}\left(w^{\prime 1}, \frac{d w^{\prime 1}}{d s}\right) Y^{\prime}, Z^{\prime}\right\rangle .
\end{aligned}
$$

Proof. If $P$ is the orthogonal projection of $T_{x} M$ on $T_{x} M^{Y}$, we know that $\nabla_{T}^{L} U=P \nabla_{T}^{M} U$, and so

$$
\nabla_{T}^{M} U=\nabla_{T} U+(I-P) \nabla_{T}^{M} U
$$


Also

$$
\nabla_{T}^{M} U=\nabla_{T} U+S(T) U
$$

Moreover, it is easy to see that if $Z \in T_{x} M$,

$$
P Z=P_{G} Z+\int_{0}^{1} P_{H} Z d s
$$

and so

$$
(I-P) Z=P_{H} Z-\int_{0}^{1} P_{H} Z d s
$$

Now if $T, U \in T M^{Y}, P_{H} \nabla_{T} U$ is constant, and so by (3.65), (3.67),

$$
(I-P) \nabla_{T}^{M} U=P_{H} S(T) U-\int_{0}^{1} P_{H} S(T) U d s,
$$

(3.60) is proved. Since $\nabla^{M}=\nabla+S,(3.61)$ is obvious. Equation (3.62) is a standard consequence of (3.60).

In particular, from (3.62), we find that

$$
\begin{aligned}
\left\langle R^{L}\left(Y^{\prime}, Z^{\prime}\right) w_{s}^{\prime 1}, \frac{d w_{s}^{\prime 1}}{d s}\right\rangle= & \int_{0}^{1}\left\langle R^{M^{0}}\left(Y^{\prime}, Z^{\prime}\right) w_{s}^{\prime 1}, d w_{s}^{\prime 1}\right\rangle \\
& -2 \int_{0}^{1}\left\langle P_{H} S\left(Y^{\prime}\right) w_{s}^{\prime 1}, P_{H} S\left(Z^{\prime}\right) d w_{s}^{\prime 1}\right\rangle .
\end{aligned}
$$

By Theorem 3.3, $S\left(Y^{\prime}\right) w^{\prime 1}, S\left(Z^{\prime}\right) d w^{\prime 1} \in T^{H} M$, and so

$$
\begin{aligned}
\left\langle R^{L}\left(Y^{\prime}, Z^{\prime}\right) w^{\prime 1}, \frac{d w^{\prime 1}}{d s}\right\rangle= & \int_{0}^{1}\left\langle R^{M^{0}}\left(Y^{\prime}, Z^{\prime}\right) w^{\prime 1}, d w^{11}\right\rangle \\
& -2 \int_{0}^{1}\left\langle S\left(Y^{\prime}\right) w^{\prime 1}, S\left(Z^{\prime}\right) d w^{\prime 1}\right\rangle .
\end{aligned}
$$

Also classically

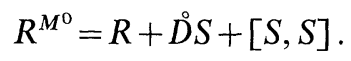

Since $D S$ sends $T G$ in $T^{H} M^{0}$, we find from (3.71) that (3.70) is also equal to

$$
\begin{gathered}
\int_{0}^{1}\left\langle R\left(Y^{\prime}, Z^{\prime}\right) w^{\prime 1}, d w^{\prime 1}\right\rangle+\int_{0}^{1}\left\langle S\left(Y^{\prime}\right) w^{\prime 1}, S\left(Z^{\prime}\right) d w^{\prime 1}\right\rangle-\int_{0}^{1}\left\langle S\left(Z^{\prime}\right) w^{\prime 1}, S\left(Y^{\prime}\right) d w^{\prime 1}\right\rangle \\
-2 \int_{0}^{1}\left\langle S\left(Y^{\prime}\right) w^{\prime 1}, S\left(Z^{\prime}\right) d w^{\prime 1}\right\rangle=\int_{0}^{1}\left\langle R\left(Y^{\prime}, Z^{\prime}\right) w^{\prime 1}, d w^{\prime 1}\right\rangle .
\end{gathered}
$$

Similarly, by (3.62), we have

$$
\begin{aligned}
\left\langle R^{L}\left(w^{\prime 1}, \frac{d w^{\prime}}{d s}\right) Y^{\prime}, Z^{\prime}\right\rangle= & \int_{0}^{1}\left\langle R^{M^{0}}\left(w^{1}, d w^{\prime 1}\right) Y^{\prime}, Z^{\prime}\right\rangle \\
& -\int_{0}^{1}\left\langle P_{H} S\left(w^{\prime 1}\right) Y^{\prime}, P_{H} S\left(d w^{1}\right) Z^{\prime}\right\rangle d s \\
& +\int_{0}^{1}\left\langle P_{H} S\left(d w^{\prime 1}\right) Y^{\prime}, P_{H} S\left(w^{1}\right) Z^{\prime}\right\rangle d s .
\end{aligned}
$$


Moreover, by Theorem 3.3, $T\left(w_{s}^{\prime 1}, \frac{d w_{s}^{\prime 1}}{d s}\right)=0$. Using (3.71), we have

$$
\begin{aligned}
\int_{0}^{1}\left\langle R^{M^{0}}\left(w^{\prime 1}, d w^{\prime 1}\right) Y^{\prime}, Z^{\prime}\right\rangle= & \int_{0}^{1}\left\langle R\left(w^{1}, d w^{\prime 1}\right) Y^{\prime}, Z^{\prime}\right\rangle+2 \int_{0}^{1}\left\langle\nabla_{w^{\prime}} S\left(d w^{\prime 1}\right) Y^{\prime}, Z^{\prime}\right\rangle \\
& +2 \int_{0}^{1}\left\langle S\left(w^{\prime 1}\right) Y^{\prime}, S\left(d w^{\prime 1}\right) Z^{\prime}\right\rangle .
\end{aligned}
$$

Using (3.73), (3.74), we find

$$
\begin{aligned}
\left\langle R^{L}\left(w^{\prime 1}, d w^{\prime 1}\right) Y^{\prime}, Z^{\prime}\right\rangle= & \int_{0}^{1}\left\langle R\left(w^{\prime 1}, d w^{\prime 1}\right) Y^{\prime}, Z^{\prime}\right\rangle+2 \int_{0}^{1}\left\langle\nabla_{w^{\prime 1}} S\left(d w^{\prime 1}\right) Y^{\prime}, Z^{\prime}\right\rangle \\
& -2 \int_{0}^{1}\left\langle P_{G} S\left(d w^{\prime 1}\right) Y^{\prime}, P_{G} S\left(d w^{\prime 1}\right) Z^{\prime}\right\rangle .
\end{aligned}
$$

Finally, since $R^{L}$ is a Levi-Civita curvature tensor

$$
\left\langle R^{L}\left(Y^{\prime}, Z^{\prime}\right) w^{\prime 1}, \frac{d w^{\prime 1}}{d s}\right\rangle=\left\langle R^{L}\left(w^{\prime 1}, \frac{d w^{\prime 1}}{d s}\right) Y^{\prime}, Z^{\prime}\right\rangle \text {. }
$$

The theorem is proved.

The analogy of Proof No. 1 of Theorem 1.9 with Proof No. 1 of Theorem 3.16 should now be clear: in particular, equality (3.42) is still a form of (1.34).

Finally, we still want to give a differential geometric interpretation of formula (3.34). Let $x$. be a loop in $M^{Y}$. Let $\frac{D}{D s}, \frac{D^{0}}{D S}$ be the covariant differentiation operators for the connections $\nabla, \nabla^{M^{0}}$ along $x$. Then by Theorem 2.2, if $Y, Z \in T M^{Y}$

$$
\frac{d X(Y, Z)}{2}=\int_{0}^{1}\left\langle\frac{D^{0} Y}{D s}, Z\right\rangle d s=\int_{0}^{1}\left\langle\frac{D Y}{D s}, Z\right\rangle d s+\int_{0}^{1}\langle S(d x) Y, Z\rangle .
$$

To integrate formally against the fiber $C$ the differential form exp $-\frac{\left(d+i_{X}\right) X}{2}$, we must select the terms of maximal (even and still infinite) degree in the vertical Grassmann variables. Now $\int_{0}^{1}\left\langle\frac{D Y}{D S}, Z\right\rangle d s$ only contains such terms. This is not the case for the second term in (3.77). Indeed, if $P_{G} Y_{0}=P_{G} Y_{1}=P_{G} Z_{0}=P_{G} Z_{1}=0$, we write the right-hand side of (3.77) as follows:

$$
\begin{aligned}
& \int_{0}^{1}\left\langle\frac{D Y}{D S}, Z\right\rangle d s+\int_{0}^{1}\langle S(d x) Y, Z\rangle \\
& =\int_{0}^{1}\left\langle\frac{D}{D s}\left[P_{G} Y_{s}+P_{G} \tau_{s}^{0} \int_{0}^{s} \tau_{0}^{h} S\left(d x_{h}\right) P_{H} Y\right], P_{G} Z_{s}+P_{G} \tau_{s}^{0} \int_{0}^{s} \tau_{0}^{h^{\prime}} S\left(d x_{h^{\prime}}\right) P_{H} Z\right\rangle d s \\
& \quad+\int_{0}^{1}\left\langle S(d x) P^{H} Y, P^{H} Z\right\rangle-\int_{0 \leqq h \leqq h^{\prime} \leqq 1}\left\langle P_{G} \tau_{0}^{h} S\left(d x_{h}\right) P_{H} Z, P_{G} \tau_{0}^{h^{\prime}} S\left(d x_{h^{\prime}}\right) P_{H} Y\right\rangle .
\end{aligned}
$$


When integrating along the fiber $C$, in the first term in the right-hand side of (3.78), terms like $P_{G} \tau_{s}^{0} \int_{0}^{s} \tau_{0}^{h} S\left(d x_{s}\right) P_{H} Y$ are obviously killed. On the contrary in the righthand side of (3.78), the last term, which only contains the horizontal $P_{H} Z, P_{H} Z^{\prime}$ remains alive.

Formula (3.34), which comes from operator theory, should now have a clearer interpretation, in the light of (3.58) and (3.78).

The introduction of superconnections, superalgebras in operator theory has now a natural interpretation on the loop space: it is by this way that operator theory "understands" the rather innocuous operation of integrating along the fiber (in the loop space) terms which mix horizontal and vertical Grassmann variables.

Acknowledgements. The author is grateful to a referee for very helpful comments and suggestions.

\section{References}

1. Alvarez-Gaumé, L.: Supersymmetry and the Atiyah-Singer Index Theorem. Commun. Math. Phys. 90, 161-173 (1983)

2. Atiyah, M.F.: Circular symmetry and stationary phase approximation. In: Proceedings of the conference in honor of L. Schwartz. Paris: Astérisque 1985 (to appear)

3. Atiyah, M.F., Bott, R.: A Lefschetz fixed point formula for elliptic complexes. I. Ann. Math. 86, 374-407 (1967); II. 88, 451-491 (1968)

4. Atiyah, M.F., Bott, R.: The moment map and equivariant cohomology. Topology 23, 1-28 (1984)

5. Atiyah, M.F., Bott, R., Patodi, V.K.: On the heat equation and the Index Theorem. Invent. Math. 19, 279-330 (1973)

6. Atiyah, M.F., Singer, I.M.: The index of elliptic operators. I. Ann. Math. 87, 484-530 (1968); III. 87, 546-604 (1968)

7. Atiyah, M.F., Singer, I.M.: The index of elliptic operators. IV. Ann. Math. 93, 119-138 (1971)

8. Berline, N., Vergne, M.: A computation of the equivariant index of the Dirac operator (to appear)

9. Berline, N., Vergne, M.: Zéros d'un champ de vecteurs et classes caractéristiques équivariantes. Duke Math. J. 50, 539-549 (1983)

10. Bismut, J.-M.: Large deviations and the Malliavin calculus. Progress in Math. No. 45. Basel: Birkhäuser 1984

11. Bismut, J.-M.: Transformations différentiables du mouvement Brownien. In: Proceedings of the conference in honor of L. Schwartz. Paris: Astérisque 1985 (to appear)

12. Bismut, J.-M.: The Atiyah-Singer theorems: a probabilistic approach. I. J. Funct. Anal. 57, 56-99 (1984); II. 57, 329-348 (1984)

13. Bismut, J.-M.: Index theorem and equivariant cohomology on the loop space. Commun. Math. Phys. 98, 213-237 (1985)

14. Bismut, J.-M.: The infinitesimal Lefschetz formulas: a heat equation proof. J. Funct. Anal. 62 , 435-457 (1985)

15. Bismut, J.-M.: The index theorem for families of Dirac operators: two heat equation proofs. Invent. Math. (to appear)

16. Bismut, J.-M., Michel, D.: Diffusions conditionnelles. I. J. Funct. Anal. 44, 174-211 (1981); II. Générateur conditionnel. Application au filtrage. 45, 272-292 (1982)

17. Bott, R., Tu, L.H.: Differential forms in algebraic topology. Graduate texts in Math., Vol. 82. Berlin, Heidelberg, New York: Springer 1982 
18. Duistermaat, J.J., Heckman, G.: On the variation of the cohomology of the reduced phase space. Invent. Math. 69, 259-268 (1982); Addendum 72, 153-158 (1983)

19. Friedan, D., Windey, H.: Supersymmetric derivation of the Atiyah-Singer index and the Chiral anomaly. Nucl. Phys. B 235, 395-416 (1984)

20. Getzler, E.: Pseudodifferential operators on supermanifolds and the Atiyah-Singer Index Theorem. Commun. Math. Phys. 92, 163-178 (1983)

21. Getzler, E.: A short proof of the Atiyah-Singer Index Theorem. Topology (to appear)

22. Lichnerowicz, A.: Spineurs harmoniques. C.R. Acad. Sci. Paris Ser. I 257, 7-9 (1963)

23. Mathai, V., Quillen, D.: Superconnections, Thom classes and equivariant differential forms (to appear)

24. McKean, H., Singer, I.M.: Curvature and the eigenvalues of the Laplacian. J. Differ. Geom. 1, 43-69 (1967)

25. Quillen, D.: Superconnections and the Chern character. Topology 24, 89-95 (1985)

26. Witten, E.: Supersymmetry and Morse theory. J. Diff. Geom. 17, 661-692 (1982)

27. Berline, N., Vergne, M.: The equivariant Index and Kirillov's character formula. Trans. Am. Math. Soc. (to appear)

Communicated by A. Jaffe

Received April 12, 1985; in revised form July 19, 1985 